\title{
Supporting Information to: Consistent Parameter Estimation for LASSO and Approximate Message Passing
}

\author{
Ali Mousavi \\ Arian Maleki \\ ali.mousavi@rice.edu arian@stat.columbia.edu \\ Richard G. Baraniuk \\ richb@rice.edu
}

\begin{abstract}
This document includes properties of risk of the soft-thresholding function, the proofs of some of our main theorems, simulation results, and a detailed description of the simulation whose results were reported in the main text.
\end{abstract}

\section{A Background}

\section{A.1 Quasiconvex functions}

Here, we briefly mention several properties of quasi-convex functions (see Section 3.4 of $[1])$. The following theorem is a key element in our proofs.

Theorem A.1. [1] A continuous function $f: \mathbb{R} \rightarrow \mathbb{R}$ is quasiconvex if and only if at least one of the following conditions holds: (1) $f$ is non-decreasing. (2) $f$ is non-increasing. (3) There is a point $c$ in the domain of $f$ such that for $t<c$ $f$ is non-increasing and for $t \geq c$, it is non-decreasing.

The following simple lemma shows that shifting and scaling preserve quasiconvexity.

Lemma A.2. Let $a$ and $b>0$ be two fixed numbers. Then $f(x)$ is a quasiconvex function if and only if $g(x)=a+b f(x)$ is a quasi-convex function.

Proof. First, assume that $f(x)$ is quasi-convex. Then, according to the definition of the quasi-convexity we can write

$$
\begin{aligned}
g(\alpha x+((1-\alpha) y) & =a+b f(\alpha x+((1-\alpha) y) \leq a+b \max (f(x), f(y)) \\
& =\max (a+b f(x), a+b f(y))=\max (g(x), g(y)) .
\end{aligned}
$$


Hence, $g(x)$ is quasi-convex as well. On the other hand, suppose that $g(x)$ is quasi-convex. Then, according to the definition, we can write

$$
\begin{aligned}
f(\alpha x+((1-\alpha) y) & =\frac{g(\alpha x+((1-\alpha) y)-a}{b} \leq \frac{\max (g(x), g(y))-a}{b} \\
& \leq \max \left(\frac{g(x)-a}{b}, \frac{g(y)-a}{b}\right) \leq \max (f(x), f(y)) .
\end{aligned}
$$

Therefore, $f(x)$ is quasi-convex as well.

\section{A.2 Risk of the soft thresholding function}

In this section we review some of the basic results (some of which have been proved elsewhere) that will be used in our paper. Let

$$
\Psi\left(\sigma^{2}\right) \triangleq \sigma_{w}^{2}+\frac{1}{\delta} \mathbb{E}_{B, Z}\left[(\eta(B+\sigma Z ; \chi \sigma)-B)^{2}\right],
$$

where $B \sim p_{\beta}$ and $Z \sim N(0,1)$ are two independent random variables. Note that $\Psi$ is a function of $\left(\delta, \chi, \sigma_{w}^{2}\right)$. We assume that all of these parameters are fixed and $\Psi$ is only a function of $\sigma^{2}$.

Lemma A.3. [2] $\Psi\left(\sigma^{2}\right)$ is a concave function of $\sigma^{2}$.

One major implication of this theorem that is related to the fixed points of $\Psi\left(\sigma^{2}\right)$ is summarized in the next lemma.

Lemma A.4. [3] For $\sigma_{w}^{2}>0, \Psi$ has a unique fixed point.

Another interesting result that will play a crucial role below is the quasiconvexity of the risk of soft thresholding in terms of the threshold. Let $\mu$ be a random variable with distribution $\mu \sim G$ independent of $Z \sim N(0,1)$ and define $r(\tau, G) \triangleq \mathbb{E}_{\mu, Z}\left[(\eta(\mu+Z ; \tau)-\mu)^{2}\right]$. We claim that $r(\tau, G)$ is a quasiconvex function of $\tau$.

Lemma A.5. $r(\tau ; G)$ is a quasi-convex function of $\tau$. Furthermore, if $\mathbb{P}(\mu=$ $0) \neq 1$, the function is bowl-shaped.

Proof. In order to prove this result we use Theorem A.1. It is straightforward to prove that $r(\tau, G)$ is a differentiable function of $\tau$. This derivative is shown in Figure 1. According to Theorem A.1, we have to prove that the derivative either has no sign change or has one sign change from negative to positive. Note that if we had $\frac{\partial^{2} r(\tau ; G)}{\partial \tau^{2}} \geq 0$ this would immediately show that the function is convex and hence also quasi-convex. However, this is not true here. Figure 1 shows $\frac{\partial}{\partial \tau} r(\tau ; G)$ as a function of $\tau$. As is clear from the figure, the derivative is not an increasing function, and hence we should not expect the second derivative to always be positive.

Instead we will show that the ratio $V(\tau ; G) \triangleq \frac{\frac{\partial}{\partial \tau} r(\tau, G)}{\frac{\partial}{\partial \tau} r\left(\tau, \delta_{0}\right) \mid}$ is strictly increasing in $\tau \in[0, \infty)$ where $\delta_{0}$ as a point mass at zero. Once we prove this, we can conclude that $V(\tau, \mu)$ will have at most one sign change, and since $\left|\frac{\partial}{\partial \tau} r\left(\tau, \delta_{0}\right)\right|$ is always positive we can conclude that $\frac{\partial r(\tau, G)}{\partial \tau}$ will have at most one sign change. 


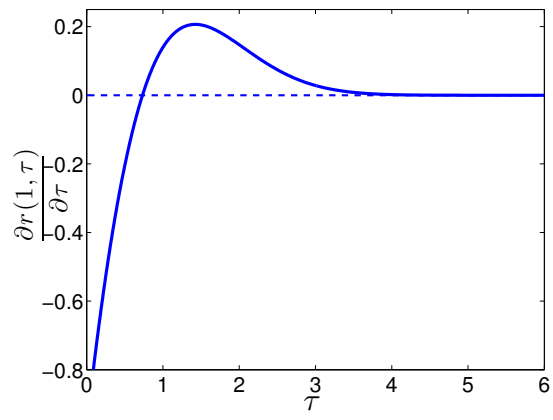

Figure 1: The derivative of $\frac{\partial}{\partial \tau} r(\mu, \tau)$ as a function of $\tau$ for $\mu=1$ and $0<\tau<\infty$. Note that the derivative of the risk has only one sign change. Below that point the derivative is negative and above of that point is positive (even though it converges to zero as $\tau \rightarrow \infty$ ). Hence we expect the risk to be quasi-convex.

According to Theorem A.1 this completes the proof of quasi-convexity. Therefore, our main goal in the rest of the proof is to show that $V(\tau, G)$ is strictly increasing. We have

$$
\begin{aligned}
& r(\tau, G)= \mathbb{E}_{\mu, Z}\left[(\eta(\mu+Z ; \tau)-\mu)^{2}\right] \\
&=\mathbb{E}_{\mu, Z}\left[(Z-\tau)^{2} \mathbb{I}(\mu+Z \geq \tau)+(Z+\tau)^{2} \mathbb{I}(\mu+Z \leq-\tau)\right. \\
&\left.\quad+\mu^{2} \mathbb{I}(|\mu+Z|<\tau)\right] .
\end{aligned}
$$

Therefore,

$$
\begin{aligned}
& \frac{\partial r(\tau, G)}{\partial \tau}=\mathbb{E}_{\mu, Z}\left[-2(Z-\tau) \mathbb{I}(\mu+Z \geq \tau)-(Z-\tau)^{2} \delta(\mu+Z-\tau)\right. \\
& +2(Z+\tau) \mathbb{I}(\mu+Z \leq-\tau)-(Z+\tau)^{2} \delta(\tau+\mu+Z) \\
& \left.+\mu^{2} \delta(\tau-|\mu+Z|)\right] \\
& =\mathbb{E}_{\mu, Z}[2(Z+\tau) \mathbb{I}(\mu+Z \leq-\tau)-2(Z-\tau) \mathbb{I}(\mu+Z \geq \tau)] \\
& =\underbrace{2 \mathbb{E}_{\mu}\left[\int_{-\infty}^{-(\mu+\tau)}(z+\tau) \phi(z) d z\right]}_{\Gamma_{1}}-\underbrace{2 \mathbb{E}_{\mu}\left[\int_{\tau-\mu}^{\infty}(z-\tau) \phi(z) d z\right]}_{\Gamma_{2}},
\end{aligned}
$$

where $\phi$ denotes the probability density function of the standard normal random variable. Changing the integral variables to $w=z+\tau$ in $\Gamma_{1}$ and $w=z-\tau$ in $\Gamma_{2}$ results in

$$
\frac{\partial r(\tau, G)}{\partial \tau}=2 \mathbb{E}_{\mu}\left[\int_{-\infty}^{-\mu} w \phi(w-\tau) d w\right]+2 \mathbb{E}_{\mu}\left[\int_{-\infty}^{\mu} w \phi(w-\tau) d w\right] .
$$

Consequently, we can write

$$
V(\tau ; G)=\frac{2 \mathbb{E}_{\mu}\left[\int_{-\infty}^{-\mu} w \phi(w-\tau) d w\right]+2 \mathbb{E}_{\mu}\left[\int_{-\infty}^{\mu} w \phi(w-\tau) d w\right]}{4 \int_{-\infty}^{0}|w| \phi(w-\tau) d w}
$$




$$
\begin{aligned}
= & \frac{2 \mathbb{E}_{\mu}\left[\int_{-\infty}^{0} w \phi(w-\tau) d w-\int_{-\mu}^{0} w \phi(w-\tau) d w\right]}{4 \int_{-\infty}^{0}|w| \phi(w-\tau) d w} \\
& +\frac{2 \mathbb{E}_{\mu}\left[\int_{-\infty}^{0} w \phi(w-\tau) d w+\int_{0}^{\mu} w \phi(w-\tau) d w\right]}{4 \int_{-\infty}^{0}|w| \phi(w-\tau) d w} \\
= & \frac{\mathbb{E}_{\mu}\left[\int_{-\mu}^{\mu}|w| \phi(w-\tau) d w\right]}{2 \int_{-\infty}^{0}|w| \phi(w-\tau) d w}-1 .
\end{aligned}
$$

Let $R(\tau, G) \triangleq \frac{\mathbb{E}_{\mu}\left[\int_{-\mu}^{\mu}|w| \phi(w-\tau) d w\right]}{\int_{-\infty}^{0}|w| \phi(w-\tau) d w}$. Taking the derivative of $R(\tau, G)$ with respect to $\tau$ gives

$$
\begin{aligned}
& \frac{\partial R(\tau, G)}{\partial \tau}=\frac{\mathbb{E}_{\mu}\left[\int_{-\mu}^{\mu}(w-\tau)|w| \phi(w-\tau) d w\right] \int_{-\infty}^{0}|w| \phi(w-\tau) d w}{\left(\int_{-\infty}^{0}|w| \phi(w-\tau) d w\right)^{2}} \\
& -\frac{\mathbb{E}_{\mu}\left[\int_{-\mu}^{\mu}|w| \phi(w-\tau) d w\right] \int_{-\infty}^{0}(w-\tau)|w| \phi(w-\tau) d w}{\left(\int_{-\infty}^{0}|w| \phi(w-\tau) d w\right)^{2}} \\
& =\frac{\overbrace{\mathbb{E}_{\mu}\left[\int_{-\mu}^{\mu} w|w| \phi(w-\tau) d w\right]}^{\Gamma_{3}} \int_{-\infty}^{0}|w| \phi(w-\tau) d w}{\left(\int_{-\infty}^{0}|w| \phi(w-\tau) d w\right)^{2}} \\
& +\frac{\overbrace{\mathbb{E}_{\mu}\left[\int_{-\mu}^{\mu}|w| \phi(w-\tau) d w\right] \int_{0}^{\infty} w|w| \phi(w+\tau) d w}^{\Gamma_{4}}}{\left(\int_{-\infty}^{0}|w| \phi(w-\tau) d w\right)^{2}} .
\end{aligned}
$$

$\Gamma_{3}$ can be simplified to

$$
\Gamma_{3}=\mathbb{E}_{\mu}\left[\int_{0}^{|\mu|} w^{2}(\phi(w-\tau)-\phi(w+\tau)) d w\right]>0 .
$$

Therefore, since $\Gamma_{4}>0$, we have $\frac{\partial R(\tau, G)}{\partial \tau}>0$ which proves that $R(\tau, G)$ is an increasing function. This, in addition to the fact that $V(0 ; G)<0$, completes the proof of quasi-convexity.

According to Theorem A.1, combining the facts that $\frac{\partial V(\tau, G)}{\partial \tau} \geq 0$ and $V(0, G)<0$ proves the quasi-convexity of the risk. However, this does not mean that the risk function is bowl-shaped (See Definition ??). In fact, the risk function is bowl-shaped if sign-change happens at certain value of $\tau$. We would now like to prove that the zero crossing in fact happens if $G \neq \delta_{0}$ (as mentioned before $\delta_{0}$ denotes the distribution of a point mass at zero). 
Lemma A.6. The risk of the soft thresholding function satisfies $\left.\frac{\partial r(\tau, G)}{\partial \tau}\right|_{\tau=0}$ $<0$.

Proof. We have

$$
\left.\frac{\partial r(\tau, G)}{\partial \tau}\right|_{\tau=0}=2 \mathbb{E}_{\mu}\left[\int_{-\infty}^{-\mu} z \phi(z) d z-\int_{-\mu}^{\infty} z \phi(z) d z\right]=2 \mathbb{E}_{\mu}\left[-\frac{2 e^{\frac{-\mu^{2}}{2}}}{\sqrt{2 \pi}}\right]<0 .
$$

If we prove that for large enough $\hat{\tau}$ we have $\left.\frac{\partial r(\tau, G)}{\partial \tau}\right|_{\tau=\hat{\tau}}>0$, then we can conclude that $\frac{\partial r(\tau, G)}{\partial \tau}$ has at least one zero-crossing. On the other hand, according to Lemma A.5, $\frac{\partial r(\tau, G)}{\partial \tau}$ has at most one zero crossing. Therefore, we would actually prove that $\frac{\partial r(\tau, G)}{\partial \tau}$ has exactly one zero-crossing and, consequently, the risk of soft-thresholding function is a bowl-shaped function. We require the following two lemmas in the proof of our main claim, which is summarized in Proposition A.8.

Lemma A.7. Suppose that $\mathbb{P}(\mu \neq 0) \neq 0$. Then, there exists an $\epsilon_{0}>0$ such that $\mathbb{P}\left(|\mu|>\epsilon_{0}\right)>0$.

The proof is trivial and hence is skipped here.

Proposition A.8. If $\mathbb{P}(\mu \neq 0) \neq 0$. Then $\left.\frac{\partial r(\tau, G)}{\partial \tau}\right|_{\tau=\hat{\tau}}>0$ for the large values of $\hat{\tau}$ that make (12) positive.

Proof. Without loss of generality and for simplicity of notation, we assume that $\mathbb{P}(\mu \geq 0)=1$. Using the same change of variable technique as in $(5)$, we have

$$
\begin{aligned}
\frac{\partial r(\tau, G)}{\partial \tau}= & 2 \mathbb{E}_{\mu}\left[\int_{-\infty}^{-\mu} w \phi(w-\tau) d w\right]+2 \mathbb{E}_{\mu}\left[\int_{-\infty}^{\mu} w \phi(w-\tau) d w\right] \\
= & 2 \mathbb{E}_{\mu}\left[\int_{-\infty}^{-\mu} w \phi(w-\tau) d w+\int_{-\infty}^{0} w \phi(w-\tau) d w\right] \\
& +2 \mathbb{E}_{\mu}\left[\int_{0}^{\mu} w \phi(w-\tau) d w\right] \\
\geq & 4 \underbrace{\mathbb{E}_{\mu}[\underbrace{\mathbb{E}_{\mu}\left[\int_{0}^{\mu} w \phi(w-\tau) d w\right]}_{\Gamma_{2}}}_{\Gamma_{1}\left[\int_{-\infty}^{0} w \phi(w-\tau) d w\right]} .
\end{aligned}
$$

Note that $\Gamma_{1}<0$, and $\Gamma_{2}>0$. Our goal is to show that for large values of $\tau$, $\Gamma_{2}>\left|\Gamma_{1}\right|$. To achieve this goal, we find an upper bound for $\left|\Gamma_{1}\right|$ and a lower bound for $\Gamma_{2}$. Simplifying $\Gamma_{1}$ gives

$$
\begin{aligned}
\Gamma_{1} & =\mathbb{E}_{\mu}\left[\int_{-\infty}^{0} w \phi(w-\tau) d w\right]=\frac{2 e^{\frac{-\tau^{2}}{2}}}{\sqrt{2 \pi}} \int_{-\infty}^{0} w e^{\frac{-w^{2}}{2}+w \tau} d w \\
& \geq \frac{2 e^{\frac{-\tau^{2}}{2}}}{\sqrt{2 \pi}} \int_{-\infty}^{0} w e^{\frac{-w^{2}}{2}} d w=-\frac{e^{\frac{-\tau^{2}}{2}}}{\sqrt{2 \pi}} \int_{-\infty}^{0}|w| e^{\frac{-w^{2}}{2}} d w \geq-\sqrt{\frac{2}{\pi}} e^{\frac{-\tau^{2}}{2}} .
\end{aligned}
$$


Therefore, it is sufficient to prove that $\Gamma_{2}>\sqrt{\frac{2}{\pi}} e^{\frac{-\tau^{2}}{2}}$. To achieve this goal, we first use $\epsilon_{0}$ as introduced in Lemma A.7; hence, we can obtain a lower bound for $\Gamma_{2}$ as:

$$
\begin{aligned}
\Gamma_{2} & =\mathbb{E}_{\mu}\left[\int_{0}^{\mu} w \phi(w-\tau) d w\right] \geq \mathbb{P}\left(\mu>\epsilon_{0}\right) \int_{0}^{\epsilon_{0}} w \phi(w-\tau) d w \\
& =\mathbb{P}\left(\mu>\epsilon_{0}\right) \frac{e^{\frac{-\tau^{2}}{2}}}{\sqrt{2 \pi}} \int_{0}^{\epsilon_{0}} w e^{\frac{-w^{2}}{2}+w \tau} d w \geq \mathbb{P}\left(\mu>\epsilon_{0}\right) \frac{e^{\frac{-\tau^{2}}{2}-\frac{\epsilon_{0}^{2}}{2}}}{\sqrt{2 \pi}} \int_{0}^{\epsilon_{0}} w e^{w \tau} d w \\
& =\mathbb{P}\left(\mu>\epsilon_{0}\right) \frac{e^{\frac{-\tau^{2}}{2}-\frac{\epsilon_{0}^{2}}{2}}}{\sqrt{2 \pi}}\left(\frac{\epsilon_{0} \mathrm{e}^{\epsilon_{0} \tau}}{\tau}-\frac{\mathrm{e}^{\epsilon_{0} \tau}}{\tau^{2}}+\frac{1}{\tau^{2}}\right)
\end{aligned}
$$

Define $c_{1} \triangleq \sqrt{\frac{2}{\pi}}$ and $c_{2} \triangleq \mathbb{P}\left(\mu>\epsilon_{0}\right) \frac{\mathrm{e}^{-\epsilon_{0}^{2} / 2}}{\sqrt{2 \pi}}$. Combining (10) and (11) gives

$$
\Gamma_{1}+\Gamma_{2} \geq \underbrace{\left(c_{2} \frac{\epsilon_{0} e^{\epsilon_{0} \tau}}{\tau}-c_{2} \frac{\mathrm{e}^{\epsilon_{0} \tau}}{\tau^{2}}+\frac{c_{2}}{\tau^{2}}+c_{1}\right)}_{\Lambda} e^{\frac{-\tau^{2}}{2}} .
$$

Clearly, for large values of $\tau$, the first term of $\Lambda$ is dominant, and hence $\Lambda$ is positive. Therefore, the proof is complete.

Combining Corollary A.6, Proposition A.8, and the fact that $\frac{\partial r(\tau, G)}{\partial \tau}$ has at most one zero-crossing proves that $r(G, \tau)$ is a quasi-convex and bowl-shaped function (see Definition ??).

Proposition A.9. $r(G, \tau)$ is a strictly decreasing function of $\tau$ for the case $G=\delta_{0}$.

Proof. We can write

$$
\begin{aligned}
r\left(\delta_{0}, \tau\right) & =\mathbb{E}\left[\eta^{2}(Z ; \tau)\right]=\mathbb{E}\left[(Z-\tau)^{2} \mathbb{I}(Z>\tau)+(Z+\tau)^{2} \mathbb{I}(Z<-\tau)\right] \\
& =\int_{\tau}^{\infty}(z-\tau)^{2} \phi(z) d z+\int_{-\infty}^{-\tau}(z+\tau)^{2} \phi(z) d z
\end{aligned}
$$

Therefore, if we take the derivative with respect to $\tau$, we have $\frac{d r\left(\delta_{0}, \tau\right)}{d \tau}=$ $-4 \int_{\tau}^{\infty} z \phi(z) d z<0$.

\section{B Proof of Proposition 3.8}

\section{B.1 Roadmap of the proof}

Since the proof of this theorem is long we describe the steps of the proof below.

1. We consider the AMP algorithm in which the threshold is set to $\chi \sigma^{t}$ at every iteration. We show that for every value of $\lambda$ there is a unique value of $\chi$ such that the solution of AMP has the same MSE as the solution of LASSO and vice versa. Hence, we conclude that if $\lambda_{\text {opt }}$ is the best choice of the regularization parameter for LASSO (that minimizes MSE), then $\chi_{\text {opt }}$ the corresponding value of $\chi$ is the best value that can be picked in AMP (when the threshold value at iteration $t$ is $\chi \sigma^{t}$ ). Clearly the other 
direction is also true, i.e., if $\chi_{\text {opt }}$ is the best value of the threshold for AMP, then the corresponding $\lambda$ will be the optimal value of the regularization parameter for LASSO.

2. In the second step we connect two versions of AMP: (i) AMP with threshold $\chi_{\text {opt }} \sigma^{t}$ and (ii) optimal AMP with threshold $\tau^{*, t}$ defined in Definition ??. We prove that the final solutions of these two AMP algorithms have the same mean square error. Note that at any iteration these two thresholding policies may lead to different estimates. In fact, the AMP algorithm that employs $\tau^{*, 1}, \tau^{*, 2}, \ldots$ converges to its final solution faster. However, they eventually converge to solutions with the same MSE.

These two steps are proved in Sections B.2 and B.3 respectively.

\section{B.2 Proof of Step 1}

According to Theorem ?? the solution of LASSO satisfies:

$$
\left.\lim _{p \rightarrow \infty} \frac{1}{p}\left\|\hat{\beta}^{\lambda}(p)-\beta_{o}(p)\right\|_{2}^{2}=\mathbb{E}_{B, W}(\eta(B+\hat{\sigma} W ; \chi \hat{\sigma})-B)\right)^{2},
$$

where $\hat{\sigma}$ and $\chi$ satisfy the following equations with $\sigma_{w}$ being the variance of the input noise:

$$
\begin{aligned}
\hat{\sigma}^{2} & =\sigma_{w}^{2}+\frac{1}{\delta} \mathbb{E}_{B, W}\left[(\eta(B+\hat{\sigma} W ; \chi \hat{\sigma})-B)^{2}\right], \\
\lambda & =\chi \hat{\sigma}\left(1-\frac{1}{\delta} \mathbb{P}(|B+\hat{\sigma} W|>\chi \hat{\sigma})\right) .
\end{aligned}
$$

Define $\lambda_{\text {opt }}$ as $\lambda_{\text {opt }} \triangleq \arg \min _{\lambda} \lim _{p \rightarrow \infty} \frac{1}{p}\left\|\hat{\beta}^{\lambda}(p)-\beta_{o}(p)\right\|_{2}^{2}$. This is the value of $\lambda$ that minimizes the asymptotic MSE. Let $\hat{\sigma}_{\text {opt }}$ and $\chi_{\text {opt }}$ denote the values of $(\hat{\sigma}, \chi)$ that satisfy $(15)$ with $\lambda_{\text {opt }}$. Suppose that we run the AMP algorithm with thresholding policy $\chi_{o p t} \sigma^{t}$. According to Theorem ?? the fixed point of this AMP algorithm will satisfy

where

$$
\left.\lim _{t \rightarrow \infty} \lim _{p \rightarrow \infty} \frac{1}{p}\left\|\hat{\beta}^{t}(p)-\beta_{o}(p)\right\|_{2}^{2}=\mathbb{E}_{B, W}\left(\eta\left(B+\hat{\sigma} W ; \chi_{o p t} \hat{\sigma}\right)-B\right)\right)^{2},
$$

$$
\hat{\sigma}^{2}=\sigma_{w}^{2}+\frac{1}{\delta} \mathbb{E}_{B, W}\left[(\eta(B+\hat{\sigma} W ; \chi \hat{\sigma})-B)^{2}\right] .
$$

According to Lemma A.4, (17) has a unique fixed point. Hence, the MSE of AMP with threshold $\chi_{o p t} \sigma^{t}$ is the same as the MSE of LASSO with optimal threshold parameter $\lambda$. As the next step we would like to show that if we use another threshold of the form $\chi \sigma^{t}$ in AMP, it will lead to larger MSE. Suppose that $\bar{\chi}$ leads to a smaller asymptotic MSE in AMP. Define $\bar{\sigma}$ as the fixed point of $\sigma_{w}^{2}+\frac{1}{\delta} \mathbb{E}_{B, W}\left[(\eta(B+\hat{\sigma} W ; \bar{\chi} \hat{\sigma})-B)^{2}\right]$. Then define $\bar{\lambda} \triangleq \bar{\chi} \bar{\sigma}\left(1-\frac{1}{\delta} \mathbb{P}(|B+\bar{\sigma} W|>\bar{\chi} \bar{\sigma})\right)$. Suppose that we use $\bar{\lambda}$ in LASSO, then according to Theorem ?? the MSE of LASSO will be the same as MSE of AMP with threshold $\bar{\chi} \sigma^{t}$ that is lower than the threshold of LASSO for $\lambda_{o p t}$. This contradiction confirms that we cannot find $\bar{\chi}$ to reduce the final asymptotic MSE of AMP. 


\section{B.3 Proof of Step 2}

Suppose that $\left(\sigma^{t}, \tau^{*, t}\right)$ converge to $\sigma_{\infty}, \tau_{\infty}$ in the optimal AMP algorithm.

Our claim is that $\sigma_{\infty}$ is the same as $\hat{\sigma}_{\text {opt }}$, discussed in the last section. By the definition of $\tau^{*, t}$ we have $\sigma_{\infty} \leq \hat{\sigma}_{\text {opt }}$. Hence, we need to prove that $\sigma_{\infty} \geq \hat{\sigma}_{\text {opt }}$. Define $\chi_{\infty} \triangleq \frac{\tau_{\infty}}{\sigma_{\infty}}$. Suppose that we run AMP with thresholding policy $\chi_{\infty} \sigma^{t}$. Then the fixed point of this version of AMP satisfies

$$
\hat{\sigma}^{2}=\sigma_{w}^{2}+\frac{1}{\delta} \mathbb{E}_{B, W}\left[\left(\eta\left(B+\hat{\sigma} W ; \chi_{\infty} \hat{\sigma}\right)-B\right)^{2}\right] .
$$

Note that this equation has a solution at $\hat{\sigma}=\sigma_{\infty}$. That is because $\chi_{\infty} \sigma_{\infty}=\tau_{\infty}$ and (18) becomes the same as the fixed point equation for the optimal AMP. Secondly, note that according to Lemma A.4, (18) has a unique fixed point. Therefore, AMP with threshold $\chi_{\infty} \sigma^{t}$ will converge to this unique fixed point. Since by construction the MSE of AMP with threshold $\chi_{\text {opt }} \sigma^{t}$ is smaller than AMP with threshold $\chi_{\infty} \sigma^{t}$, we conclude that $\sigma_{\infty} \geq \hat{\sigma}_{\text {opt }}$. This completes the proof of the second step.

\section{Proof of Proposition 3.9}

We first mention a preliminary result that will be used in our proof.

Theorem C.1. [4] Let $X_{i j} \stackrel{i i d}{\sim} N(0,1 / n)$. Then, the largest singular value of $X$ converges to $1+\sqrt{p / n}$ almost surely.

The proof of Proposition 3.9 is based on an induction argument. Let $\tilde{\beta}^{t}(p)$ and $\tilde{z}^{t}(p)$ denote the estimates we obtain from AMP with threshold parameter $\hat{\tau}^{t}$. Also, let $\beta_{*}^{t}(p)$ and $z_{*}^{t}(p)$ denote the estimates of AMP with threshold parameters $\tau_{*}^{t}$. We run induction on the following statements simultaneously:

$$
\begin{aligned}
& \lim _{p \rightarrow \infty} \frac{\left\|\tilde{\beta}^{t}-\beta_{*}^{t}\right\|_{2}^{2}}{p}=0, \\
& \lim _{p \rightarrow \infty} \frac{\left\|\tilde{z}^{t}-z_{*}^{t}\right\|_{2}^{2}}{p}=0,
\end{aligned}
$$

in probability. Since the base of the induction is straightforward, for the sake of brevity we skip it and only prove that the inductive step holds, i.e., we assume (19) and prove

$$
\begin{aligned}
& \lim _{p \rightarrow \infty} \frac{\left\|\tilde{\beta}^{t+1}(p)-\beta_{*}^{t+1}(p)\right\|_{2}^{2}}{p}=0, \\
& \lim _{p \rightarrow \infty} \frac{\left\|\tilde{z}^{t+1}(p)-z_{*}^{t+1}(p)\right\|_{2}^{2}}{p}=0,
\end{aligned}
$$

in probability. We start with the proof of (20). We have

$$
\frac{\left\|\tilde{\beta}^{t+1}(p)-\beta_{*}^{t+1}(p)\right\|_{2}}{\sqrt{p}}=\frac{\left\|\eta\left(\tilde{\beta}^{t}+X^{*} \tilde{z}^{t} ; \hat{\tau}^{t}\right)-\eta\left(\beta_{*}^{t}+X^{*} z_{*}^{t} ; \tau_{*}^{t}\right)\right\|_{2}}{\sqrt{p}}
$$




$$
\begin{aligned}
= & \frac{\left\|\eta\left(\tilde{\beta}^{t}+X^{*} \tilde{z}^{t} ; \hat{\tau}^{t}\right)-\eta\left(\tilde{\beta}^{t}+X^{*} \tilde{z}^{t} ; \tau_{*}^{t}\right)\right\|_{2}}{\sqrt{p}} \\
& +\frac{\left\|\eta\left(\tilde{\beta}^{t}+X^{*} \tilde{z}^{t} ; \tau_{*}^{t}\right)-\eta\left(\beta_{*}^{t}+X^{*} z_{*}^{t} ; \tau_{*}^{t}\right)\right\|_{2}}{\sqrt{p}} \\
\leq & \left|\hat{\tau}^{t}-\tau_{*}^{t}\right|+\frac{\left\|\tilde{\beta}^{t}+X^{*} \tilde{z}^{t}-\beta_{*}^{t}-X^{*} z_{*}^{t}\right\|_{2}}{\sqrt{p}} \\
\leq & \left|\hat{\tau}^{t}-\tau_{*}^{t}\right|+\frac{\left\|\tilde{\beta}^{t}-\beta_{*}^{t}\right\|_{2}}{\sqrt{p}}+\sigma_{\max }\left(X^{*}\right) \frac{\left\|\tilde{z}^{t}-z_{*}^{t}\right\|_{2}}{\sqrt{p}} \rightarrow 0,
\end{aligned}
$$

in probability, where the last step uses the assumptions of the inductive step, and the facts that $\hat{\tau}^{t} \rightarrow \tau_{*}^{t}$ by the assumption of the theorem. Note that $\sigma_{\max }\left(X^{*}\right)$ is bounded with probability converging to one according to Theorem C.1 .

The second step is to prove (21). We have

$$
\begin{aligned}
& \frac{\left\|\tilde{z}^{t+1}(p)-z_{*}^{t+1}(p)\right\|_{2}}{\sqrt{p}}=\frac{\left\|X \tilde{\beta}^{t}-X \beta_{*}^{t}-\right\| \tilde{\beta}^{t}\left\|_{0} \tilde{z}^{t} / n+\right\| \beta_{*}^{t}\left\|_{0} z_{*}^{t} / n\right\|_{2}}{\sqrt{p}} \\
\leq & \frac{\left\|X \tilde{\beta}^{t}-X \beta_{*}^{t}\right\|_{2}}{\sqrt{p}}+\frac{\|\| \tilde{\beta}^{t}\left\|_{0} \tilde{z}^{t} / n-\right\| \beta_{*}^{t}\left\|_{0} z_{*}^{t} / n\right\|_{2}}{\sqrt{p}} \\
\leq & \frac{\left\|X \tilde{\beta}^{t}-X \beta_{*}^{t}\right\|_{2}}{\sqrt{p}}+\frac{\|\| \tilde{\beta}^{t}\left\|_{0} \tilde{z}^{t} / n-\right\| \tilde{\beta}^{t}\left\|_{0} z_{*}^{t} / n\right\|_{2}}{\sqrt{p}} \\
& +\frac{\|\| \tilde{\beta}^{t}\left\|_{0} z_{*}^{t} / n-\right\| \beta_{*}^{t}\left\|_{0} z_{*}^{t} / n\right\|_{2}}{\sqrt{p}} .
\end{aligned}
$$

Similar to what we have done so far, it is straightforward to show that the first two terms converge to zero in probability. Hence, we only focus on the last term. In particular, we show that

$$
\text { | }|| \tilde{\beta}^{t}\left\|_{0} / n-\right\| \beta_{*}{ }^{t} \|_{0} / n \mid \rightarrow 0,
$$

We should mention that since $\left\|z_{*}\right\|_{2}^{2} / n \rightarrow\left(\sigma^{t}\right)^{2}$ according to Lemma 1 of [5], (24) will imply (23). As discussed in Section 2.3, we have

$$
\left\|\beta_{*}{ }^{t}\right\|_{0} / p \rightarrow E_{B, W}\left[\left|\eta\left(B+\sigma^{t} W ; \tau_{*}^{t}\right)\right|>0\right],
$$

in probability. Furthermore, for every bounded Lipschitz function $\psi$ with Lipschitz constant $L$ we have

$$
\begin{aligned}
& \left|\frac{1}{p} \sum_{i=1}^{p} \psi\left(\tilde{\beta}_{i}^{t}\right)-\frac{1}{p} \sum_{i=1}^{p} \psi\left(\beta_{*, i}^{t}\right)\right| \\
& \leq \frac{1}{p} \sum_{i=1}^{p}\left|\psi\left(\tilde{\beta}_{i}^{t}\right)-\psi\left(\beta_{*, i}^{t}\right)\right| \\
& \leq \frac{L}{p} \sum_{i=1}^{p}\left|\tilde{\beta}_{i}^{t}-\beta_{*, i}^{t}\right| \\
& \leq \frac{L\left\|\beta_{*}^{t}-\tilde{\beta}^{t}\right\|_{2}}{\sqrt{p}},
\end{aligned}
$$

where the last equality is a result of the Cauchy-Schwartz inequality. Since 
$\left\|\beta_{*}^{t}-\tilde{\beta}^{t}\right\|_{2} / \sqrt{p} \rightarrow 0$, we conclude that $\left|\frac{1}{p} \sum_{i=1}^{p} \psi\left(\tilde{\beta}_{i}^{t}\right)-\frac{1}{p} \sum_{i=1}^{p} \psi\left(\beta_{*, i}^{t}\right)\right| \rightarrow 0$ in probability. According to Theorem 2.4 we know that

$$
\frac{1}{p} \sum_{i=1}^{p} \psi\left(\beta_{*, i}^{t}\right) \rightarrow E_{B, W}\left[\psi\left(\eta\left(B+\sigma^{t} W ; \tau_{*}^{t}\right)\right)\right],
$$

in probability. Hence, for every Lipschitz function $\psi$

$$
\frac{1}{p} \sum_{i=1}^{p} \psi\left(\tilde{\beta}_{i}^{t}\right) \rightarrow E_{B, W}\left[\psi\left(\eta\left(B+\sigma^{t} W ; \tau_{*}^{t}\right)\right)\right]
$$

in probability. Hence, the empirical distribution of $\tilde{\beta}^{t}$ converges to the distribution of $\eta\left(B+\sigma^{t} W ; \tau_{*}^{t}\right)$. Note that since $W$ has a Gaussian distribution, the distribution of $B+\sigma^{t} W$ is absolutely continuous everywhere. Therefore,

$$
\left\|\tilde{\beta}^{t}\right\|_{0} / p \rightarrow E_{B, W}\left[\left|\eta\left(B+\sigma^{t} W ; \tau_{*}^{t}\right)\right|>0\right]=\mathbb{P}\left(\left|B+\sigma^{t} W\right|>\tau_{*}^{t}\right) .
$$

Combining (25) and (27) proves (24) and hence completes the paper.

\section{Proof of Theorem 3.10}

First note that, according to Theorem ??, we have $\lim _{N \rightarrow \infty} \frac{1}{N} \sum_{i=1}^{N} \mathbb{I}\left(\hat{\beta}_{i}^{\lambda}(N) \neq\right.$ $0)=\mathbb{P}(|\eta(B+\hat{\sigma} Z ; \chi \hat{\sigma})|>0)$, where $(\hat{\sigma}, \lambda, \chi)$ satisfy the following equations:

$$
\begin{aligned}
& \hat{\sigma}^{2}=\sigma_{z}^{2}+\frac{1}{\delta} \mathbb{E}_{B, Z}\left[(\eta(B+\hat{\sigma} Z ; \chi \hat{\sigma})-B)^{2}\right], \\
& \lambda=\chi \hat{\sigma}\left(1-\frac{1}{\delta} \mathbb{P}(|B+\hat{\sigma} Z|>\chi \hat{\sigma})\right) .
\end{aligned}
$$

Therefore, we have to prove that $\frac{d \mathbb{P}(|\eta(B+\hat{\sigma} Z ; \chi \hat{\sigma})|>0)}{d \lambda}<0$. The difficulty of this task is clear from $(28):(\chi, \hat{\sigma}, \lambda)$ are complicated functions of each other whose explicit formulations are not known. Using the chain rule we have

$$
\frac{d}{d \lambda} \mathbb{P}(|\eta(B+\hat{\sigma} Z ; \chi \hat{\sigma})|>0)=\frac{d}{d \chi} \mathbb{P}(|\eta(B+\hat{\sigma} Z ; \chi \hat{\sigma})|>0) \frac{d \chi}{d \lambda} .
$$

This expression enables us to break the proof into the following two simpler parts: (i) We prove that $\left(\frac{d}{d \chi} \mathbb{P}(|\eta(B+\hat{\sigma} Z ; \chi \hat{\sigma})|>0)\right)<0$. (ii) We prove that $\frac{d \chi}{d \lambda}>0$. Combining these two results with (29) completes the proof.

Lemma D.1. Let $(\chi, \hat{\sigma})$ satisfy (28). Then $\left(\frac{d}{d \chi} \mathbb{P}(|\eta(B+\hat{\sigma} Z ; \chi \hat{\sigma})|>0)\right)<0$.

Proof. Since $|\eta(B+\hat{\sigma} Z ; \chi \hat{\sigma})|>0$ if and only if $\left|\frac{B}{\hat{\sigma}}+Z\right|>\chi$, it is sufficient to prove $\frac{d}{d \chi} \mathbb{P}\left(\left|\frac{B}{\hat{\sigma}}+Z\right|>\chi\right)<0$. We have

$$
\frac{d}{d \chi} \mathbb{P}\left(\left|\frac{B}{\hat{\sigma}}+Z\right|>\chi\right)=\frac{\partial}{\partial \hat{\sigma}} \mathbb{P}\left(\left|\frac{B}{\hat{\sigma}}+Z\right|>\chi\right) \frac{d \hat{\sigma}}{d \chi}+\frac{\partial}{\partial \chi} \mathbb{P}\left(\left|\frac{B}{\hat{\sigma}}+Z\right|>\chi\right) .
$$

The rest of the proof has four main steps: (i) Calculation of $\frac{\partial}{\partial \hat{\sigma}} \mathbb{P}\left(\left|\frac{B}{\hat{\sigma}}+Z\right|>\chi\right)$. (ii) Calculation of $\frac{\partial}{\partial \chi} \mathbb{P}\left(\left|\frac{B}{\hat{\sigma}}+Z\right|>\chi\right)$. (iii) Calculation of $\frac{d \hat{\sigma}}{d \chi}$. Finally, (iv) Plugging the results of the above three steps in (30) and proving that

$$
\left(\frac{d}{d \chi} \mathbb{P}\left(\left|\frac{B}{\hat{\sigma}}+Z\right|>\chi\right)\right)<0 .
$$


Here are these four steps realized:

Step i: Calculation of $\frac{\partial}{\partial \hat{\sigma}} \mathbb{P}\left(\left|\frac{B}{\hat{\sigma}}+Z\right|>\chi\right)$ : Simple algebra leads us to

$$
\begin{aligned}
\frac{\partial}{\partial \hat{\sigma}} \mathbb{P}\left(\left|\frac{B}{\hat{\sigma}}+Z\right| \geq \chi\right) & =\mathbb{E}_{B}\left[\frac{\partial}{\partial \hat{\sigma}} \mathbb{E}_{Z}\left[\mathbb{I}\left(\left|\frac{B}{\hat{\sigma}}+Z\right| \geq \chi\right) \mid B\right]\right] \\
& =\mathbb{E}_{B}\left[\frac{\partial}{\partial \hat{\sigma}}\left(\int_{\chi-\frac{B}{\hat{\sigma}}}^{\infty} \phi(z) d z+\int_{-\infty}^{-\chi-\frac{B}{\hat{\sigma}}} \phi(z) d z\right)\right] \\
& =\mathbb{E}_{B}\left[\frac{B}{\hat{\sigma}^{2}} \phi\left(\frac{B}{\hat{\sigma}}+\chi\right)-\frac{B}{\hat{\sigma}^{2}} \phi\left(\frac{B}{\hat{\sigma}}-\chi\right)\right] .
\end{aligned}
$$

Step ii: Calculation of $\frac{\partial}{\partial \chi} \mathbb{P}\left(\left|\frac{B}{\hat{\sigma}}+Z\right|>\chi\right)$ : Similar to Step 1, we have

$$
\begin{aligned}
\frac{\partial}{\partial \chi} \mathbb{P}\left(\left|\frac{B}{\hat{\sigma}}+Z\right| \geq \chi\right) & =\mathbb{E}_{B}\left[\frac{\partial}{\partial \chi} \mathbb{E}_{Z}\left[\mathbb{I}\left(\left|\frac{B}{\hat{\sigma}}+Z\right| \geq \chi\right) \mid B\right]\right] \\
& =\mathbb{E}_{B}\left[\frac{\partial}{\partial \chi}\left(\int_{\chi-\frac{B}{\hat{\sigma}}}^{\infty} \phi(z) d z+\int_{-\infty}^{-\chi-\frac{B}{\hat{\sigma}}} \phi(z) d z\right)\right] \\
& =\mathbb{E}_{B}\left[-\phi\left(\frac{B}{\hat{\sigma}}+\chi\right)-\phi\left(\frac{B}{\hat{\sigma}}-\chi\right)\right] .
\end{aligned}
$$

Step iii: Calculation of $\frac{d \hat{\sigma}}{d \chi}$ : We can rewrite (28) as

$$
\delta=\frac{\delta \sigma_{z}^{2}}{\hat{\sigma}^{2}}+\mathbb{E}_{B, Z}\left[\left(\eta\left(\frac{B}{\hat{\sigma}}+Z ; \chi\right)-\frac{B}{\hat{\sigma}}\right)^{2}\right] .
$$

Taking the derivative of both sides of (33) with respect to $\chi$ yields

$$
\begin{aligned}
0= & \frac{-2 \delta \sigma_{z}^{2}}{\hat{\sigma}^{3}} \frac{d \hat{\sigma}}{d \chi}+\frac{d}{d \chi} \mathbb{E}_{B, Z}\left[\left(\eta\left(\frac{B}{\hat{\sigma}}+Z ; \chi\right)-\frac{B}{\hat{\sigma}}\right)^{2}\right] \\
= & \frac{-2 \delta \sigma_{z}^{2}}{\hat{\sigma}^{3}} \frac{d \hat{\sigma}}{d \chi}+\frac{\partial}{\partial \chi} \mathbb{E}_{B, Z}\left[\left(\eta\left(\frac{B}{\hat{\sigma}}+Z ; \chi\right)-\frac{B}{\hat{\sigma}}\right)^{2}\right] \\
& +\frac{\partial}{\partial \hat{\sigma}} \mathbb{E}_{B, Z}\left[\left(\eta\left(\frac{B}{\hat{\sigma}}+Z ; \chi\right)-\frac{B}{\hat{\sigma}}\right)^{2}\right] \frac{d \hat{\sigma}}{d \chi} \\
= & \frac{-2 \delta \sigma_{z}^{2}}{\hat{\sigma}^{3}} \frac{d \hat{\sigma}}{d \chi}+2 \mathbb{E}_{B, Z}\left[\left(\eta\left(\frac{B}{\hat{\sigma}}+Z ; \chi\right)-\frac{B}{\hat{\sigma}}\right) \frac{\partial}{\partial \chi}\left(\eta\left(\frac{B}{\hat{\sigma}}+Z ; \chi\right)-\frac{B}{\hat{\sigma}}\right)\right] \\
& +2 \mathbb{E}_{B, Z}\left[\left(\eta\left(\frac{B}{\hat{\sigma}}+Z ; \chi\right)-\frac{B}{\hat{\sigma}}\right) \frac{\partial}{\partial \hat{\sigma}}\left(\eta\left(\frac{B}{\hat{\sigma}}+Z ; \chi\right)-\frac{B}{\hat{\sigma}}\right)\right] \frac{d \hat{\sigma}}{d \chi} \\
= & \frac{-2 \delta \sigma_{z}^{2}}{\hat{\sigma}^{3}} \frac{d \hat{\sigma}}{d \chi}+2 \mathbb{E}_{B, Z}\left[( \eta ( \frac { B } { \hat { \sigma } } + Z ; \chi ) - \frac { B } { \hat { \sigma } } ) \left(-\mathbb{I}\left(\frac{B}{\hat{\sigma}}+Z>\chi\right)\right.\right. \\
& \quad\left(\frac{B}{\hat{\sigma}}+Z-\chi\right) \delta\left(\frac{B}{\hat{\sigma}}+Z-\chi\right) \\
& \left.\left.+\mathbb{I}\left(\frac{B}{\hat{\sigma}}+Z<-\chi\right)-\left(\frac{B}{\hat{\sigma}}+Z+\chi\right) \delta\left(\frac{B}{\hat{\sigma}}+Z+\chi\right)\right)\right]
\end{aligned}
$$




$$
\begin{aligned}
&+2 \mathbb{E}_{B, Z}[\left(\eta\left(\frac{B}{\hat{\sigma}}+Z ; \chi\right)-\frac{B}{\hat{\sigma}}\right)\left(\left(\frac{-B}{\hat{\sigma}^{2}}\right) \mathbb{I}\left(\frac{B}{\hat{\sigma}}+Z>\chi\right)\right. \\
&+\left(\frac{B}{\hat{\sigma}}+Z-\chi\right) \delta\left(\frac{B}{\hat{\sigma}}+Z-\chi\right)\left(\frac{-B}{\hat{\sigma}^{2}}\right) \\
&+\left(\frac{-B}{\hat{\sigma}^{2}}\right) \mathbb{I}\left(\frac{B}{\hat{\sigma}}+Z<-\chi\right) \\
&\left.\left.+\left(\frac{B}{\hat{\sigma}}+Z+\chi\right) \delta\left(\frac{B}{\hat{\sigma}}+Z+\chi\right)\left(\frac{B}{\hat{\sigma}^{2}}\right)\right)\right] \frac{d \hat{\sigma}}{d \chi} \\
&=\frac{-\delta \sigma_{z}^{2}}{\hat{\sigma}^{3}} \frac{d \hat{\sigma}}{d \chi}+ \mathbb{E}_{B, Z}\left[\frac{B}{\hat{\sigma}^{2}}\left(\eta\left(\frac{B}{\hat{\sigma}}+Z ; \chi\right)-\frac{B}{\hat{\sigma}}\right)\left(\mathbb{I}\left(\left|\frac{B}{\hat{\sigma}}+Z\right|<\chi\right)\right)\right] \frac{d \hat{\sigma}}{d \chi} \\
&+\mathbb{E}_{B, Z}\left[\left(\eta\left(\frac{B}{\hat{\sigma}}+Z ; \chi\right)-\frac{B}{\hat{\sigma}}\right)\left(-\mathbb{I}\left(\frac{B}{\hat{\sigma}}+Z>\chi\right)+\mathbb{I}\left(\frac{B}{\hat{\sigma}}+Z<-\chi\right)\right)\right] .
\end{aligned}
$$

Therefore, we can write

$$
\begin{aligned}
\frac{d \hat{\sigma}}{d \chi} & =\frac{E_{B, Z}\left[\left(\eta\left(\frac{B}{\hat{\sigma}}+Z ; \chi\right)-\frac{B}{\hat{\sigma}}\right)\left(-\mathbb{I}\left(\frac{B}{\hat{\sigma}}+Z>\chi\right)+\mathbb{I}\left(\frac{B}{\hat{\sigma}}+Z<-\chi\right)\right)\right]}{\frac{\delta \sigma_{z}^{2}}{\hat{\sigma}^{3}}+\mathbb{E}_{B, Z}\left[\frac{B^{2}}{\hat{\sigma}^{3}}\left(\mathbb{I}\left(\left|\frac{B}{\hat{\sigma}}+Z\right|<\chi\right)\right)\right]} \\
& =\frac{\mathbb{E}_{B}\left[\int_{\chi-\frac{B}{\sigma}}^{\infty}(\chi-z) \phi(z) d z+\int_{-\infty}^{-\chi-\frac{B}{\hat{\sigma}}}(z+\chi) \phi(z) d z\right]}{\frac{\delta \sigma_{z}^{2}}{\hat{\sigma}^{3}}+\mathbb{E}_{B, Z}\left[\frac{B^{2}}{\hat{\sigma}^{3}}\left(\mathbb{I}\left(\left|\frac{B}{\hat{\sigma}}+Z\right|<\chi\right)\right)\right]} .
\end{aligned}
$$

Step iv: Proving that $\left(\frac{d}{d \chi} \mathbb{P}\left(\left|\frac{B}{\hat{\sigma}}+Z\right|>\chi\right)\right)<0$ : If we plug (35) into (30), we obtain

$$
\begin{aligned}
& \frac{d}{d \chi} \mathbb{P}\left(\left|\frac{B}{\hat{\sigma}}+Z\right|>\chi\right)=\frac{d}{d \chi} \mathbb{E}_{B, Z}\left[\mathbb{I}\left(\left|\frac{B}{\hat{\sigma}}+Z\right|>\chi\right)\right] \\
& =\frac{\partial}{\partial \chi} \mathbb{E}_{B, Z}\left[\int_{\chi-\frac{B}{\sigma}}^{\infty} \phi(z) d z+\int_{-\infty}^{-\chi-\frac{B}{\sigma}} \phi(z) d z\right] \\
& +\frac{\partial}{\partial \hat{\sigma}} \mathbb{E}_{B, Z}\left[\int_{\chi-\frac{B}{\hat{\sigma}}}^{\infty} \phi(z) d z+\int_{-\infty}^{-\chi-\frac{B}{\hat{\sigma}}} \phi(z) d z\right] \frac{d \hat{\sigma}}{d \chi} \\
& =-\overbrace{\frac{\mathbb{E}_{B, Z}\left[\phi\left(\chi-\frac{B}{\hat{\sigma}}\right)+\phi\left(\chi+\frac{B}{\hat{\sigma}}\right)\right] \frac{\delta \sigma_{z}^{2}}{\hat{\sigma}^{3}}}{{\hat{\delta \sigma_{z}^{2}}}_{\hat{\sigma}^{3}}+\mathbb{E}_{B, Z}\left[\frac{B^{2}}{\hat{\sigma}^{3}}\left(\mathbb{I}\left(\left|\frac{B}{\hat{\sigma}}+Z\right|<\chi\right)\right)\right]}}^{\Lambda_{1}} \\
& -\overbrace{\frac{\mathbb{E}_{B, Z}\left[\phi\left(\chi-\frac{B}{\hat{\sigma}}\right)+\phi\left(\chi+\frac{B}{\hat{\sigma}}\right)\right] \mathbb{E}_{B, Z}\left[\frac{B^{2}}{\hat{\sigma}^{3}}\left(\mathbb{I}\left(\left|\frac{B}{\hat{\sigma}}+Z\right|<\chi\right)\right)\right]}{\Lambda^{2} \sigma_{z}^{2}}+\mathbb{E}_{B, Z}\left[\frac{B^{2}}{\hat{\sigma}^{3}}\left(\mathbb{I}\left(\left|\frac{B}{\hat{\sigma}}+Z\right|<\chi\right)\right)\right]}^{\Lambda^{3}}
\end{aligned}
$$




$$
\begin{aligned}
& +\frac{\overbrace{\mathbb{E}_{B, Z}\left[\frac{B}{\hat{\sigma}^{2}}\left(-\phi\left(\chi-\frac{B}{\hat{\sigma}}\right)+\phi\left(\chi+\frac{B}{\hat{\sigma}}\right)\right)\right] \chi\left(\int_{\chi-\frac{B}{\hat{\sigma}}}^{\infty} \phi(z) d z+\int_{-\infty}^{-\chi-\frac{B}{\hat{\sigma}}} \phi(z) d z\right)}^{\Lambda_{3}}}{\frac{\delta \sigma_{z}^{2}}{\hat{\sigma}^{3}}+\mathbb{E}_{B, Z}\left[\frac{B^{2}}{\hat{\sigma}^{3}}\left(\mathbb{I}\left(\left|\frac{B}{\hat{\sigma}}+Z\right|<\chi\right)\right)\right]} \\
& +\frac{\overbrace{\mathbb{E}_{B, Z}\left[\frac{B}{\hat{\sigma}^{2}}\left(-\phi\left(\chi-\frac{B}{\hat{\sigma}}\right)+\phi\left(\chi+\frac{B}{\hat{\sigma}}\right)\right)\right]\left(-\int_{\chi-\frac{B}{\hat{\sigma}}}^{\infty} z \phi(z) d z+\int_{-\infty}^{-\chi-\frac{B}{\hat{\sigma}}} z \phi(z) d z\right)}^{\Lambda_{4}}}{\frac{\delta \sigma_{z}^{2}}{\hat{\sigma}^{3}}+\mathbb{E}_{B, Z}\left[\frac{B^{2}}{\hat{\sigma}^{3}}\left(\mathbb{I}\left(\left|\frac{B}{\hat{\sigma}}+Z\right|<\chi\right)\right)\right]} .
\end{aligned}
$$

Considering the terms in (36), we can write: (i) $\Lambda_{1} \geq 0$, since all the terms in $\Lambda_{1}$ are positive. (ii) $\Lambda_{3} \leq 0$, since for $B<0$ we have $\phi\left(\frac{B}{\hat{\sigma}}+\chi\right)>\phi\left(\frac{B}{\hat{\sigma}}-\chi\right)$ and for $B>0$ we have $\phi\left(\frac{B}{\hat{\sigma}}+\chi\right)<\phi\left(\frac{B}{\hat{\sigma}}-\chi\right)$. (iii) For the other two terms, $\Lambda_{2}$ and $\Lambda_{4}$, we have

$$
\begin{aligned}
& \Lambda_{2}+\Lambda_{4} \\
& =-\frac{\mathbb{E}_{B}\left[\left(\phi\left(\chi-\frac{B}{\hat{\sigma}}\right)+\phi\left(\chi+\frac{B}{\hat{\sigma}}\right)\right)\left(\frac{B}{\hat{\sigma}^{2}} \int_{-\chi-\frac{B}{\hat{\sigma}}}^{\chi-\frac{B}{\hat{\sigma}}} z \phi(z) d z+\frac{B^{2}}{\hat{\sigma}^{3}} \int_{-\chi-\frac{B}{\hat{\sigma}}}^{\chi-\frac{B}{\hat{\sigma}}} \phi(z) d z\right)\right]}{\frac{\delta \sigma_{z}^{2}}{\hat{\sigma}^{3}}+\mathbb{E}_{B, Z}\left[\frac{B^{2}}{\hat{\sigma}^{3}}\left(\mathbb{I}\left(\left|\frac{B}{\hat{\sigma}}+Z\right|<\chi\right)\right)\right]} \\
& =-\frac{\mathbb{E}_{B}\left[\left(\phi\left(\chi-\frac{B}{\hat{\sigma}}\right)+\phi\left(\chi+\frac{B}{\hat{\sigma}}\right)\right)\left(\frac{B}{\hat{\sigma}^{2}} \int_{-\chi-\frac{B}{\hat{\sigma}}}^{\chi-\frac{B}{\hat{\sigma}}}\left(z+\frac{B}{\hat{\sigma}}\right) \phi(z) d z\right)\right]}{\frac{\delta \sigma_{z}^{2}}{\hat{\sigma}^{3}}+\mathbb{E}_{B, Z}\left[\frac{B^{2}}{\hat{\sigma}^{3}}\left(\mathbb{I}\left(\left|\frac{B}{\hat{\sigma}}+Z\right|<\chi\right)\right)\right]} \\
& =-\frac{\mathbb{E}_{B}[\left(\phi\left(\chi-\frac{B}{\hat{\sigma}}\right)+\phi\left(\chi+\frac{B}{\hat{\sigma}}\right)\right) \overbrace{\left(\frac{B}{\hat{\sigma}^{2}} \int_{-\chi}^{\chi} w \phi\left(w-\frac{B}{\hat{\sigma}}\right) d z\right)}^{\Upsilon}]}{\frac{\delta \sigma_{z}^{2}}{\hat{\sigma}^{3}}+\mathbb{E}_{B, Z}\left[\frac{B^{2}}{\hat{\sigma}^{3}}\left(\mathbb{I}\left(\left|\frac{B}{\hat{\sigma}}+Z\right|<\chi\right)\right)\right]} .
\end{aligned}
$$

Regarding $\Upsilon$ in (37), for any $\epsilon \in[-\chi, 0$ ), If $B<0$ (or $B>0$ ) then

$$
\begin{gathered}
\epsilon\left(\phi\left(\epsilon-\frac{B}{\hat{\sigma}}\right)-\phi\left(-\epsilon-\frac{B}{\hat{\sigma}}\right)\right)<0 \\
\left(\operatorname{or} \epsilon\left(\phi\left(\epsilon-\frac{B}{\hat{\sigma}}\right)-\phi\left(-\epsilon-\frac{B}{\hat{\sigma}}\right)\right)>0\right) .
\end{gathered}
$$

Therefore, $\Upsilon>0$ and consequently $\Lambda_{2}+\Lambda_{4}<0$.

Putting (i), (ii), and (iii) together we conclude $\frac{d}{d \chi} \mathbb{P}\left(\left|\frac{B}{\hat{\sigma}}+Z\right|>0\right)<0$.

Lemma D.2. Let $(\chi, \hat{\sigma}, \lambda)$ satisfy (28). Then $\frac{d \lambda}{d \chi}>0$.

Proof. Taking the derivative from both sides of (28) with respect to $\chi$ yields

$$
\frac{d \lambda}{d \chi}=\underbrace{\frac{d(\chi \hat{\sigma})}{d \chi}\left(1-\frac{1}{\delta} \mathbb{P}(|B+\hat{\sigma} Z|>\chi \hat{\sigma})\right)}_{\Theta_{1}}-\underbrace{\chi \hat{\sigma} \frac{d}{d \chi} \mathbb{P}(|B+\hat{\sigma} Z|>\chi \hat{\sigma})}_{\Theta_{2}} .
$$

According to Lemma D.1, $\Theta_{2}<0$. Regarding $\Theta_{1}$, since $\lambda \geq 0$ satisfies (28), $\left(1-\frac{1}{\delta} \mathbb{P}(|B+\hat{\sigma} Z|>\chi \hat{\sigma})\right)>0$, and hence it is sufficient to prove that $\frac{d(\chi \hat{\sigma})}{d \chi} \geq 0$. 
We have

$$
\begin{aligned}
& \frac{d(\chi \hat{\sigma})}{d \chi}=\hat{\sigma}+\chi \frac{d \hat{\sigma}}{d \chi} \\
& \stackrel{(a)}{=} \hat{\sigma}+\chi \frac{E_{B, Z}\left[\left(\eta\left(\frac{B}{\hat{\sigma}}+Z ; \chi\right)-\frac{B}{\hat{\sigma}}\right)\left(-\mathbb{I}\left(\frac{B}{\hat{\sigma}}+Z>\chi\right)+\mathbb{I}\left(\frac{B}{\hat{\sigma}}+Z<-\chi\right)\right)\right]}{\frac{\delta \sigma_{z}^{2}}{\hat{\sigma}^{3}}+\mathbb{E}_{B, Z}\left[\frac{B^{2}}{\hat{\sigma}^{3}}\left(\mathbb{I}\left(\left|\frac{B}{\hat{\sigma}}+Z\right|<\chi\right)\right)\right]} \\
& =\frac{\frac{\delta \sigma_{z}^{2}}{\hat{\sigma}^{2}}+\mathbb{E}_{B, Z}\left[\frac{B^{2}}{\hat{\sigma}^{2}}\left(\mathbb{I}\left|\frac{B}{\hat{\sigma}}+Z\right|<\chi\right)\right]}{\frac{\delta \sigma_{z}^{2}}{\hat{\sigma}^{3}}+\mathbb{E}_{B, Z}\left[\frac{B^{2}}{\hat{\sigma}^{3}}\left(\mathbb{I}\left(\left|\frac{B}{\hat{\sigma}}+Z\right|<\chi\right)\right)\right]} \\
& +\frac{\mathbb{E}_{B}\left[-\int_{\chi-\frac{B}{\hat{\sigma}}}^{\infty}(z-\chi) \phi(z) d z+\int_{-\infty}^{-\frac{B}{\hat{\sigma}}-\chi}(z+\chi) \phi(z) d z\right]}{\frac{\delta \sigma_{z}^{2}}{\hat{\sigma}^{3}}+\mathbb{E}_{B, Z}\left[\frac{B^{2}}{\hat{\sigma}^{3}}\left(\mathbb{I}\left(\left|\frac{B}{\hat{\sigma}}+Z\right|<\chi\right)\right)\right]}
\end{aligned}
$$

where Equality (a) is due to (35). In order to simplify (39), we use (28) again

$$
\begin{aligned}
\delta= & \frac{\sigma_{z}^{2} \delta}{\hat{\sigma}^{2}}+\mathbb{E}_{B, Z}\left[\left(\eta\left(\frac{B}{\hat{\sigma}}+Z ; \chi\right)-\frac{B}{\hat{\sigma}}\right)^{2}\right] \\
= & \frac{\sigma_{z}^{2} \delta}{\hat{\sigma}^{2}}+\mathbb{E}_{B, Z}\left[\frac{B^{2}}{\hat{\sigma}^{2}} \mathbb{I}\left(\left|\frac{B}{\hat{\sigma}}+Z\right|<\chi\right)\right] \\
& +\mathbb{E}_{B}\left[\int_{\chi-\frac{B}{\hat{\sigma}}}^{\infty}(z-\chi)^{2} \phi(z) d z\right]+\mathbb{E}_{B}\left[\int_{-\infty}^{-\chi-\frac{B}{\sigma}}(z+\chi)^{2} \phi(z) d z\right] \\
= & \frac{\sigma_{z}^{2} \delta}{\hat{\sigma}^{2}}+\mathbb{E}_{B, Z}\left[\frac{B^{2}}{\hat{\sigma}^{2}} \mathbb{I}\left(\left|\frac{B}{\hat{\sigma}}+Z\right|<\chi\right)\right] \\
& +\beta \mathbb{E}_{B}\left[-\int_{\chi-\frac{B}{\hat{\sigma}}}^{\infty}(z-\chi) \phi(z) d z+\int_{-\infty}^{-\frac{B}{\hat{\sigma}}-\chi}(z+\chi) \phi(z) d z\right] \\
& +\mathbb{E}_{B}\left[\int_{\chi-\frac{B}{\hat{\sigma}}}^{\infty} z(z-\beta) \phi(z) d z+\int_{-\infty}^{-\frac{B}{\hat{\sigma}}-\chi} z(z+\chi) \phi(z) d z\right] .
\end{aligned}
$$

Thus, we can rewrite (39) as

$$
\begin{aligned}
\frac{d(\chi \hat{\sigma})}{d \chi}= & \frac{\delta-\mathbb{E}_{B}\left[\int_{\chi-\frac{B}{\hat{\sigma}}}^{\infty} z(z-\chi) \phi(z) d z+\int_{-\infty}^{-\frac{B}{\hat{\sigma}}-\chi} z(z+\chi) \phi(z) d z\right]}{\frac{\delta \sigma_{z}^{2}}{\hat{\sigma}^{3}}+\mathbb{E}_{B, Z}\left[\frac{B^{2}}{\hat{\sigma}^{3}}\left(\mathbb{I}\left(\left|\frac{B}{\hat{\sigma}}+Z\right|<\chi\right)\right)\right]} \\
= & \frac{\delta-\mathbb{E}_{B}\left[\int_{\chi-\frac{B}{\hat{\sigma}}}^{\infty} z^{2} \phi(z) d z+\int_{-\infty}^{-\chi \frac{B}{\hat{\sigma}}} z^{2} \phi(z) d z\right]}{\frac{\delta \sigma_{z}^{2}}{\hat{\sigma}^{3}}+\mathbb{E}_{B, Z}\left[\frac{B^{2}}{\hat{\sigma}^{3}}\left(\mathbb{I}\left(\left|\frac{B}{\hat{\sigma}}+Z\right|<\chi\right)\right)\right]} \\
& +\frac{-\chi \mathbb{E}_{B}\left[-\int_{\chi-\frac{B}{\hat{\sigma}}}^{\infty} z \phi(z) d z+\int_{-\infty}^{-\chi \frac{B}{\hat{\sigma}}} z \phi(z) d z\right]}{\frac{\delta \sigma_{z}^{2}}{\hat{\sigma}^{3}}+\mathbb{E}_{B, Z}\left[\frac{B^{2}}{\hat{\sigma}^{3}}\left(\mathbb{I}\left(\left|\frac{B}{\hat{\sigma}}+Z\right|<\chi\right)\right)\right]} \\
= & \frac{\delta-\mathbb{E}_{B}\left[\left(\chi-\frac{B}{\hat{\sigma}}\right) \phi\left(\chi-\frac{B}{\hat{\sigma}}\right)+\left(\chi+\frac{B}{\hat{\sigma}}\right) \phi\left(\chi+\frac{B}{\hat{\sigma}}\right)\right]}{\frac{\delta \sigma_{z}^{2}}{\hat{\sigma}^{3}}+\mathbb{E}_{B, Z}\left[\frac{B^{2}}{\hat{\sigma}^{3}}\left(\mathbb{I}\left(\left|\frac{B}{\hat{\sigma}}+Z\right|<\chi\right)\right)\right]}
\end{aligned}
$$




$$
\begin{aligned}
& +\frac{\mathbb{E}_{B}\left[\int_{\chi-\frac{B}{\sigma}}^{\infty} \phi(z) d z+\int_{-\infty}^{-\chi-\frac{B}{\sigma}} \phi(z) d z\right]}{\frac{\delta \sigma_{z}^{2}}{\hat{\sigma}^{3}}+\mathbb{E}_{B, Z}\left[\frac{B^{2}}{\hat{\sigma}^{3}}\left(\mathbb{I}\left(\left|\frac{B}{\hat{\sigma}}+Z\right|<\chi\right)\right)\right]} \\
& +\frac{\chi \mathbb{E}_{B}\left[\phi\left(\chi-\frac{B}{\hat{\sigma}}\right)+\phi\left(\chi+\frac{B}{\hat{\sigma}}\right)\right]}{\frac{\delta \sigma_{z}^{2}}{\hat{\sigma}^{3}}+\mathbb{E}_{B, Z}\left[\frac{B^{2}}{\hat{\sigma}^{3}}\left(\mathbb{I}\left(\left|\frac{B}{\hat{\sigma}}+Z\right|<\chi\right)\right)\right]} \\
& =\overbrace{\frac{\delta-\mathbb{E}_{B, Z}\left[\mathbb{I}\left(\left|\frac{B}{\hat{\sigma}}+Z\right|>\chi\right)\right]}{\Delta_{1}}}^{\frac{\delta \sigma_{z}^{2}}{\hat{\sigma}^{3}}+\mathbb{E}_{B, Z}\left[\frac{B^{2}}{\hat{\sigma}^{3}}\left(\mathbb{I}\left(\left|\frac{B}{\hat{\sigma}}+Z\right|<\chi\right)\right)\right]} \\
& +\overbrace{\frac{\mathbb{E}_{B}\left[\frac{B}{\hat{\sigma}}\left(\phi\left(\chi-\frac{B}{\hat{\sigma}}\right)-\phi\left(\chi+\frac{B}{\hat{\sigma}}\right)\right)\right]}{\frac{\delta \sigma_{z}^{2}}{\hat{\sigma}^{3}}+\mathbb{E}_{B, Z}\left[\frac{B^{2}}{\hat{\sigma}^{3}}\left(\mathbb{I}\left(\left|\frac{B}{\hat{\sigma}}+Z\right|<\chi\right)\right)\right]}}^{\Delta_{2}} .
\end{aligned}
$$

$\Delta_{1}>0$ due to the fact that $\lambda>0$ in (28). Furthermore, If $B<0$ (or $B>0$ ), then $\phi\left(\chi-\frac{B}{\hat{\sigma}}\right)<\phi\left(\chi+\frac{B}{\hat{\sigma}}\right) \quad\left(\right.$ or $\left.\phi\left(\chi-\frac{B}{\hat{\sigma}}\right)>\phi\left(\chi+\frac{B}{\hat{\sigma}}\right)\right)$. Therefore $\Delta_{2}>0$ and hence, $\frac{d \lambda}{d \chi}>0$. Applying

$\frac{d}{d \chi} \mathbb{P}\left(\left|\frac{B}{\hat{\sigma}}+Z\right|>0\right)<0$ and $\frac{d \lambda}{d \chi}>0$ into (29) completes the proof. Also, combining Theorem ?? and the fact that $\lambda$ is positive in (??) results in

$$
\lim _{p \rightarrow \infty} \frac{1}{p} \sum_{i} \mathbb{I}\left(\beta_{i}^{\lambda}(p) \neq 0\right) \leq \delta .
$$

\section{E Proof of Theorem 3.11}

First note that, according to Theorem ??, we have $\lim _{p \rightarrow \infty} \frac{1}{p}\left\|\hat{\beta}^{\lambda}(p)-\beta_{o}\right\|_{2}^{2}=$ $\mathbb{E}_{B, Z}\left[(\eta(B+\hat{\sigma} Z ; \chi \hat{\sigma})-B)^{2}\right]$, where $(\chi, \hat{\sigma}, \lambda)$ satisfies

$$
\begin{aligned}
& \hat{\sigma}^{2}=\sigma_{z}^{2}+\frac{1}{\delta} \mathbb{E}_{B, Z}\left[(\eta(B+\hat{\sigma} Z ; \chi \hat{\sigma})-B)^{2}\right], \\
& \lambda=\chi \hat{\sigma}\left(1-\frac{1}{\delta} \mathbb{P}(|B+\hat{\sigma} Z|>\chi \hat{\sigma})\right) .
\end{aligned}
$$

Clearly, the quasi-convexity of $\mathbb{E}\left[(\eta(B+\hat{\sigma} W ; \chi \hat{\sigma})-B)^{2}\right]$ in terms of $\lambda$ is equivalent to the quasi-convexity of $\hat{\sigma}^{2}$ in terms of $\lambda$ ( see Lemma A.2 for more information). Therefore, in the rest of the proof, our goal is to prove that $\hat{\sigma}^{2}$ is a quasi-convex function of $\lambda$.

$\hat{\sigma}^{2}$ is a differentiable function of $\lambda$. Therefore, according to Theorem A.1, $\hat{\sigma}^{2}$ is a quasi-convex function of $\lambda$ if and only if $\frac{d \hat{\sigma}^{2}}{d \lambda}$ has at most one sign change from negative to positive. We have $\frac{d \hat{\sigma}^{2}}{d \lambda}=\frac{d \hat{\sigma}^{2}}{d \chi} \frac{d \chi}{d \lambda}$. Hence, the rest of the proof involves two main steps: (i) $\frac{d \chi}{d \lambda}>0$. We have proved that this is true in Lemma D.2. (ii) $\frac{d \hat{\sigma}^{2}}{d \chi}$ has exactly one sign change from negative to positive. According 
to $(35)$ we have

$$
\frac{d \hat{\sigma}^{2}}{d \chi}=\frac{\frac{\partial}{\partial \chi} \mathbb{E}_{B, Z}\left[\left(\eta\left(\frac{B}{\hat{\sigma}}+Z ; \chi\right)-\frac{B}{\hat{\sigma}}\right)^{2}\right]}{\frac{\delta \sigma_{z}^{2}}{\hat{\sigma}^{3}}+\mathbb{E}_{B, Z}\left[\frac{B^{2}}{\hat{\sigma}^{3}}\left(\mathbb{I}\left(\left|\frac{B}{\hat{\sigma}}+Z\right|<\chi\right)\right)\right]} .
$$

As a result, the sign of $\frac{d \hat{\sigma}^{2}}{d \chi}$ is the same as sign of $\frac{\partial}{\partial \chi} \mathbb{E}\left[\left(\eta\left(\frac{B}{\hat{\sigma}}+Z ; \chi\right)-\frac{B}{\hat{\sigma}}\right)^{2}\right]$. However, this term is the derivative of the risk of the soft thresholding function with respect to its second parameter. This is what we proved in Lemma A.5. This, combined with the fact that $d \chi / d \lambda<0$, proves that $d \hat{\sigma}^{2} / d \lambda$ has exactly one sign change.

\section{F Proof of Theorem 3.14}

We first mention the following preliminary result that is used later in the proof. We use $\beta^{t}(p)$ and $\beta^{t}$ interchangeably.

Theorem F.1. Consider a standard converging sequence $\left\{\beta_{o}(p), X(p), w(p)\right\}$. If $\beta^{t}(p), z^{t}(p)$ are the estimates of $A M P$ at iteration $t$, then

almost surely.

$$
\lim _{p \rightarrow \infty} \frac{\left\|\beta^{t}(p)-\beta^{t-1}(p)\right\|_{2}^{2}}{p}=0, \text { and } \lim _{p \rightarrow \infty} \frac{\left\|z^{t}(p)-z^{t-1}(p)\right\|_{2}^{2}}{p}=0,
$$

For the proof of this result see Lemma 4.3 of [6].Now note that from the AMP iterations we have

Hence,

$$
z^{t}=y-X \beta^{t}+\frac{\left|I^{t}\right|}{n} z^{t-1}
$$

$$
z^{t}=\frac{y-X \beta^{t}}{1-\left|I^{t}\right| / n}+\frac{\left|I^{t}\right| / n}{1-\left|I^{t}\right| / n}\left(z^{t-1}-z^{t}\right) .
$$

We can employ this to conclude that if $\hat{I}^{\lambda}$ denotes the active set of the solution of LASSO, then

$$
\begin{aligned}
& \left\|\beta^{t}+X^{*} z^{t}-\hat{\beta}^{\lambda}-\frac{X^{*}\left(y-X \hat{\beta}^{\lambda}\right)}{1-\left|\hat{I}^{\lambda}\right| / n}\right\|_{2} \\
& \leq\left\|\hat{\beta}^{\lambda}-\beta^{t}\right\|_{2}+\left\|\frac{X^{*}\left(y-X \beta^{t}\right)}{1-\left|I^{t}\right| / n}-\frac{X^{*}\left(y-X \hat{\beta}^{\lambda}\right)}{1-\left|\hat{I}^{\lambda}\right| / n}\right\|_{2}+\frac{\left|I^{t}\right| / n}{1-\left|I^{t}\right| / n}\left\|z^{t-1}-z^{t}\right\|_{2} \\
& \leq\left\|\hat{\beta}^{\lambda}-\beta^{t}\right\|_{2}+\left\|\frac{X^{*}\left(X \hat{\beta}^{\lambda}-X \beta^{t}\right)}{1-\left|\hat{I}^{\lambda}\right| / n}\right\|_{2}+\left\|\frac{X^{*}\left(y-X \beta^{t}\right)}{\left.1-\left|I^{t}\right| / n\right)}-\frac{X^{*}\left(y-X \beta^{t}\right)}{1-\left|\hat{I}^{\lambda}\right| / n}\right\|_{2} \\
& +\frac{\left|I^{t}\right| / n}{1-\left|I^{t}\right| / n}\left\|z^{t-1}-z^{t}\right\|_{2} \\
& \leq\left\|\hat{\beta}^{\lambda}-\beta^{t}\right\|_{2}+\left\|\frac{X^{*}\left(X \hat{\beta}^{\lambda}-X \beta^{t}\right)}{1-\left|\hat{I}^{\lambda}\right| / n}\right\|_{2}+\left\|\frac{X^{*}\left(y-X \beta^{t}\right)}{1-\left|I^{t}\right| / n}-\frac{X^{*}\left(y-X \beta^{t}\right)}{1-\left|\hat{I}^{\lambda}\right| / n}\right\|_{2}
\end{aligned}
$$




$$
+\frac{\left|I^{t}\right| / n}{1-\left|I^{t}\right| / n}\left\|z^{t-1}-z^{t}\right\|_{2}
$$

First note that according to Theorem 3.9 for a fixed value of $\lambda>0$ the almost sure limit of $\frac{\hat{I}^{\lambda}}{n}$ is less than 1 . We use this fact to show that all these four terms converge to zero almost surely as $p \rightarrow \infty$ and then $t \rightarrow \infty$.

Here is the core of our arguments. In these arguments we assume that AMP uses $\tau^{t}=\chi \sigma^{t}$, where $\chi$ is selected such that $\lambda=\chi \hat{\sigma}(1-\mathbb{P}(|B+\hat{\sigma} W| \geq \chi \hat{\sigma}))$ with $\hat{\sigma}=\lim _{t \rightarrow \infty} \sigma^{t}$. (i) According to Theorem $2.5 \lim _{t \rightarrow \infty} \lim _{p \rightarrow \infty} \frac{1}{p} \| \hat{\beta}^{\lambda}(p)-$ $\beta^{t}(p) \|_{2}^{2}=0$ almost surely. (ii) By combining Theorem C.1 and Theorem 2.5 we conclude that $X^{*} X\left(\beta^{t}-\hat{\beta}^{\lambda}\right)$ converges to zero almost surely. (iii) According to Theorem F.1 $\| z^{t}-z^{t-1 \|_{2}}$ converges to zero almost surely. Hence, the only remaining steps are to show that $\left.|| I^{t}|/ n-| \hat{I}\right|^{\lambda} / n \mid \rightarrow 0$ almost surely and that $\left|\hat{I}^{\lambda} /\right| n$ and $\left|I^{t}\right| / n$ converge to a number below 1 . To prove these two facts, first note that according to Theorem 3.9 for every value of $\lambda, \hat{I}^{\lambda} / n$ converges to a number less than 1. Furthermore, as discussed in Remark 2.3 almost surely

$$
\frac{\left|\hat{I}^{\lambda}\right|}{p} \rightarrow \mathbb{P}(|B+\hat{\sigma} W|>\chi \hat{\sigma})<1,
$$

where $B$ and $W$ are two independent random variables with distributions $p_{\beta}$ and $N(0,1)$ respectively. Similarly, we have

$$
\frac{\left|I^{t}\right|}{p} \rightarrow \mathbb{P}\left(\left|B+\sigma^{t} W\right|>\chi \sigma^{t}\right) .
$$

Since, $\sigma^{t} \rightarrow \hat{\sigma}$, it is straightforward to see that $\lim _{t \rightarrow 0} \lim _{p \rightarrow \infty}\left|\frac{\left|I^{t}\right|}{p}-\frac{\left|\hat{I}^{\lambda}\right|}{p}\right| \rightarrow 0$ almost surely. By combining all these facts we obtain that almost surely

$$
\lim _{t \rightarrow \infty} \lim _{p \rightarrow \infty} \frac{1}{\sqrt{p}}\left\|\beta^{t}+X^{*} z^{t}-\hat{\beta}^{\lambda}+X^{*} \frac{y-X \hat{\beta}^{\lambda}}{1-\left|\hat{I}^{\lambda}\right| / n}\right\|_{2}=0 .
$$

\section{G Simulation Results}

\section{G.1 Objectives}

Our theoretical results are based on the asymptotic analysis. Asymptotic analysis is usually criticized for its lack of applicability to the medium problem sizes. In this section our goal is to evaluate the performance of our risk estimates and our tuning schemes in medium problem sizes through simulations. Our results in this section intend to answer the following questions:

1. The first question of practical importance is for what problem sizes such asymptotic analysis is useful. While addressing this question in its full generality is quite challenging, we will provide some simulation results to provide some rule of thumb for practitioners and also shed some light on the finite sample size applicability of our results.

2. Under the asymptotic settings the choice of the parameters in the risk estimate and the tuning schemes are clear. However, they are less clear in 
the medium problem sizes. In Section G.4 we show that the parameters of our algorithms can be tuned easily for medium problem sizes as well. Furthermore, we will present a parameter free version of approximate message passing that requires no tuning from the user.

3. We present some simulations on the overall performance of our parameterfree AMP algorithm and compare it with some of the other existing versions of AMP.

\section{G.2 Risk estimate and impact of sample size}

This section explores the accuracy of the risk estimate presented in (??) of the main text. We first explain how we set $h$ and $\sigma^{t}$ in (??) of the main text and then show the accuracy of the final estimate in Section G.2.3.
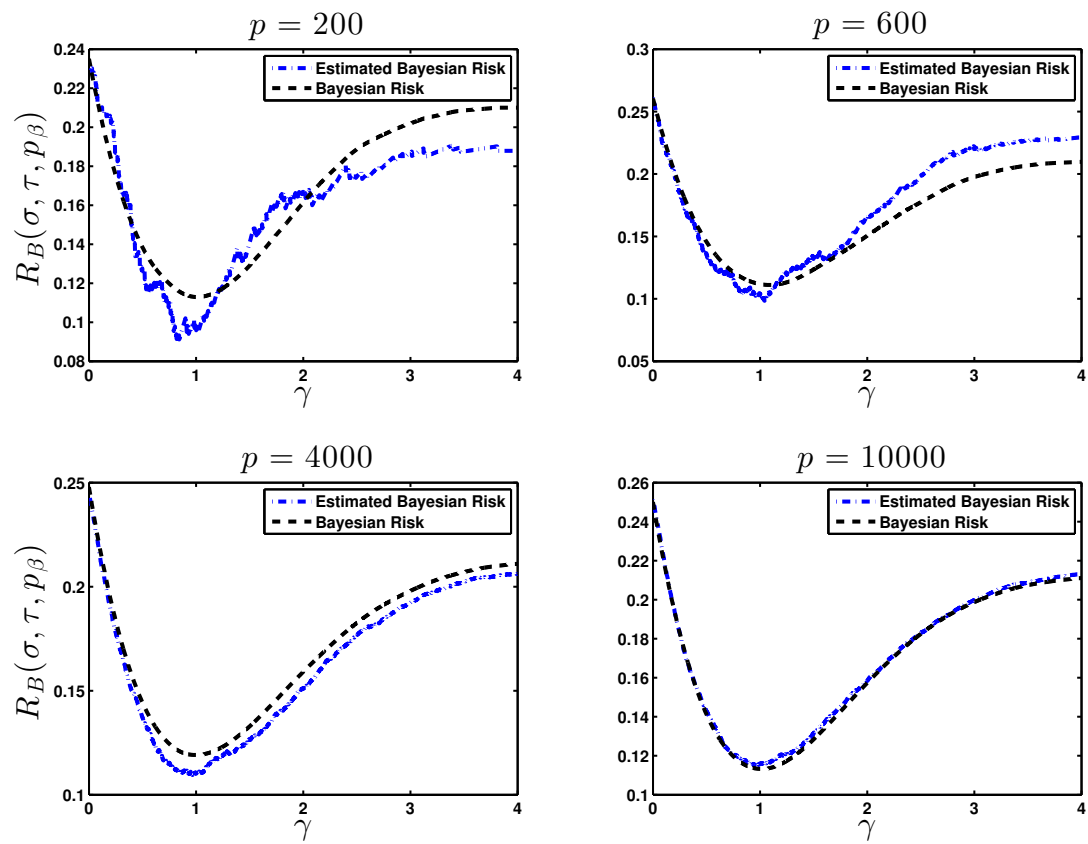

Figure 2: Performance of the risk estimator for different values of $p$ in the $1^{\text {st }}$ iteration of AMP. In this experiment $\delta=0.85, \rho=0.25$ and we consider noiseless measurements. Black curve is the Bayes risk to which our estimates will converge as $p \rightarrow \infty$. 

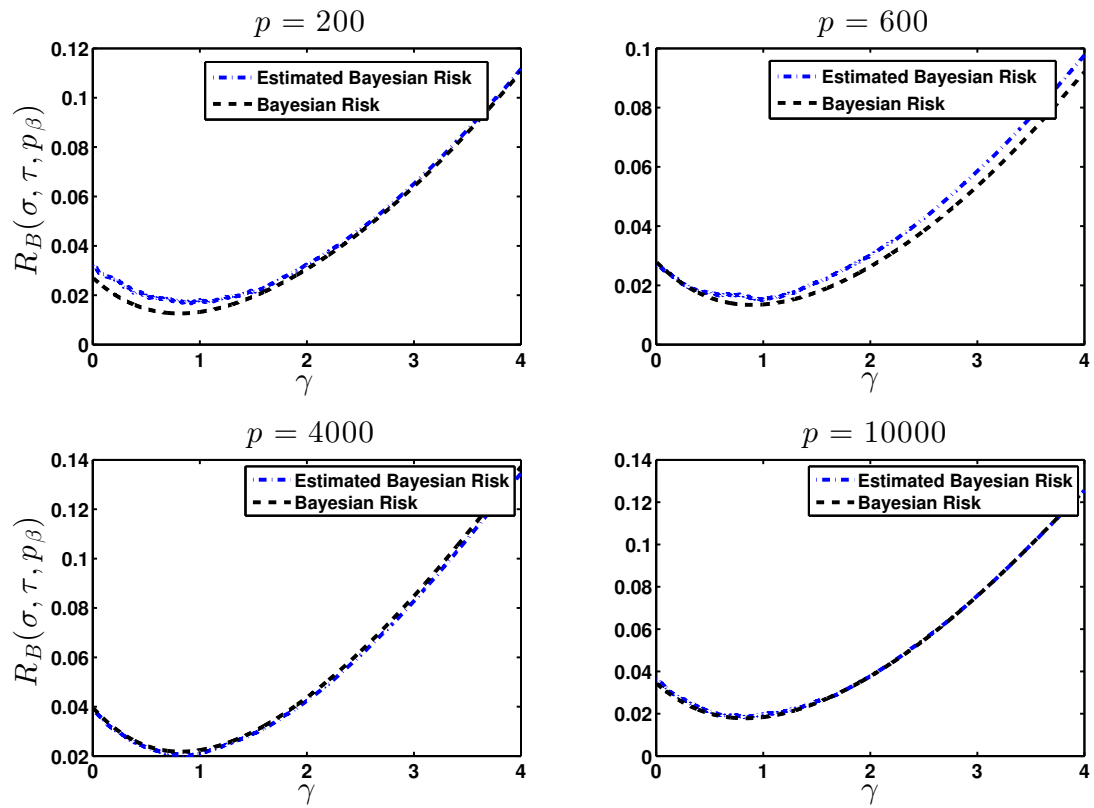

Figure 3: Performance of the risk estimator for different values of $p$ in the $10^{\text {th }}$ iteration of AMP. In this experiment $\delta=0.85, \rho=0.25$ and we consider noiseless measurements. Black curve is the Bayes risk to which our estimates will converge as $p \rightarrow \infty$.

\section{G.2.1 Smoothing parameter $h$}

The risk estimate, given in (??) of the main text, employs a Gaussian kernel with bandwidth $h$ for smoothing the soft-thresholding function. While this step is required for our theoretical analysis, our simulations suggest that the estimate is accurate even for $h=0 . h=0$ is hence used in all our simulations. We believe all our theoretical results are also true when $h=0$. However we leave the theoretical justification of this claim for future investigations.

\section{G.2.2 Estimating the noise variance}

The second issue with our risk estimate is the existence of $\sigma^{t}$ in (??) of the main text that is not known in practice. As we mentioned in Section ??, the estimate $\left(\hat{\sigma}^{t}\right)^{2}=\frac{1}{n} \sum_{i=1}^{n}\left(z_{i}^{t}\right)^{2}$ provides a consistent estimate of $\sigma^{t}$ and all the results we proved regarding the risk estimate are also accurate even if we replace $\sigma^{t}$ with $\hat{\sigma}^{t}$. In all the simulations reported here we have used the estimate of the variance instead of its actual value. 


\section{G.2.3 Impact of $p$ and $n$ on the risk estimate}

The main goal of this section is to evaluate the accuracy of the risk estimate for different values of $p$. In our simulations we have assumed that $\beta_{o}$ has $k$ non-zero coefficients, all of which have the same value $\mu$. This distribution is known to be the least favorable distribution for LASSO and AMP (with soft thresholding) [3]. The value of $\mu$ is mentioned in each simulation. We present the average risk and also the estimated risk at iterations 1 and 10 of AMP. Given the notation $\rho=k / n$ we have considered the following three cases:

1. Figure 2 and Figure 3: $p \in\{200,600,4000,10000\}, \delta=0.85$, and $\rho=0.25$, $\sigma_{w}=0, \mu=1$.

2. Figure 4 and Figure 5: $p \in\{200,600,4000,10000\}, \delta=0.85$, and $\rho=0.25$, $\sigma_{w}=0.5, \mu=1$.

3. Figure 6 and Figure 7: $p \in\{200,600,4000,10000\}, \delta=0.2$, and $\rho=0.1$, $\sigma_{w}=0.1, \mu=1$.

4. Figure 8 and Figure 9: $p \in\{10000,50000,100000,200000\}, \delta=0.01$, and $\rho=0.1, \sigma_{w}=0, \mu=100$.

5. Figure 10 and Figure 11: $p \in\{10000,50000,100000,200000\}, \delta=0.01$, and $\rho=0.1, \sigma_{w}=0.1, \mu=100$.

The results of our simulations are summarized in Figures 2 to 11. A rule of thumb that these figures suggest is that when $\delta>0.2, p>5000$ is enough. As $\delta$ decreases the estimates require $p$ to be larger. For instance, for $\delta=0.01$ $p>50000$ is required for the estimates to be accurate.

\section{G.3 The effect of dependency in the design matrix on risk estimates}

In all our theoretical analysis we assumed that the elements of $X$ are iid normal with mean zero and standard deviation $1 / n$. This assumption may be violated in many application areas. In this section we would like to show some simulation results to show that our tuning schemes return reasonably accurate estimates even when the predictors, $X_{i j}$, are weakly correlated. We will also show that as we increase the dependencies our schemes become less accurate.

Matrix $\mathcal{C} \in \mathbb{R}^{p \times p}$ in (45) shows the covariance matrix for the rows of measurement matrix $X \in \mathbb{R}^{n \times p}$. In other words, rows of measurement matrix $X$ are drawn from a multivariate normal distribution with mean zero and covariance matrix $\mathcal{C}$. Figure 12 shows the performance of AMP tuned with our proposed modified bisection method for different values of $\kappa$. It shows the result of an experiment where $p=10000, \delta=0.3, \rho=0.1$, measurements are noiseless, and we consider a point mass at 100 for the distribution of non-zero entries of $\beta_{o}$. In this setting, as Figure 12 suggests, for $\kappa$ values less than 0.2 AMP's performance is robust to covariate dependency. However, for $\kappa$ values larger than 0.3 AMP 

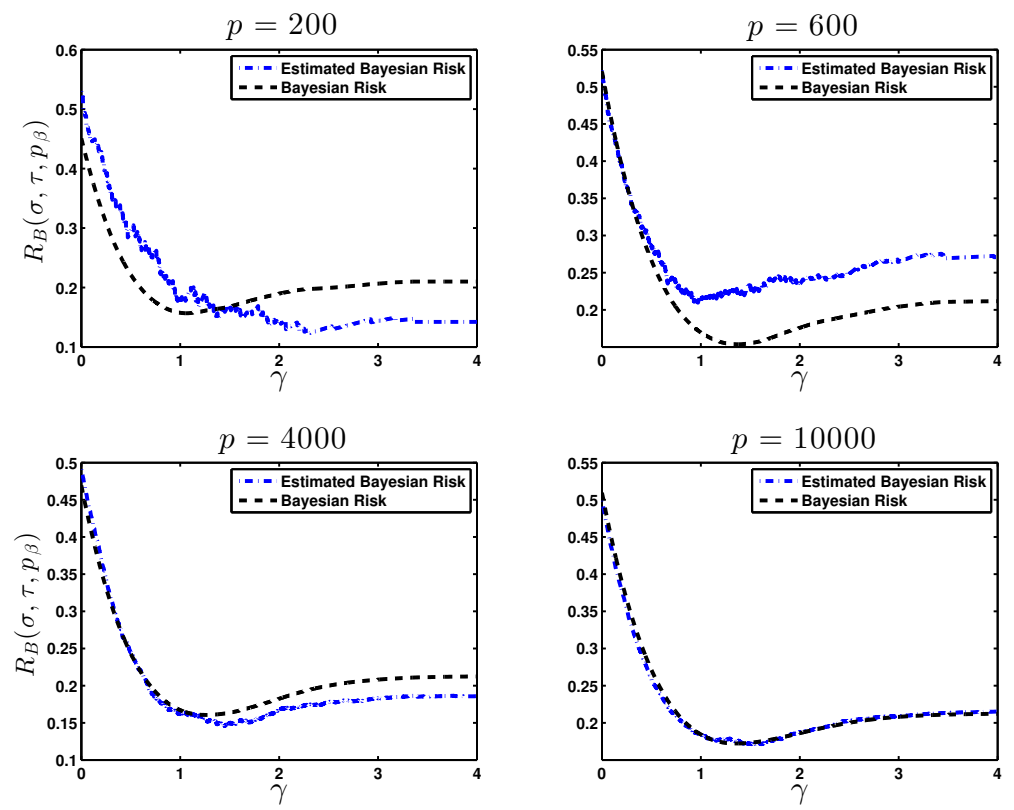

Figure 4: Performance of the risk estimator for different values of $p$ in the $1^{\text {st }}$ iteration of AMP. In this experiment $\delta=0.85, \rho=0.25$ and the standard deviation of measurement noise is 0.5. Black curve is the Bayes risk to which our estimates will converge as $p \rightarrow \infty$.

risk function starts to diverge quickly. For $\kappa=0.3$, AMP risk first converges to an MSE (0.6) close to the minimum LASSO risk $(\sim 0)$; however, it finally diverges. This phenomenon is not observed in Figure 12 since we have only included 10 iterations to compare the AMP's risk for different values of $\kappa$ before it diverges quickly for some values, e.g. $\kappa=0.4$.

$$
\mathcal{C}=\frac{1}{n} \times\left[\begin{array}{cccccc}
1 & \kappa & 0 & 0 & \ldots & 0 \\
\kappa & 1 & \kappa & 0 & \ldots & 0 \\
0 & \kappa & 1 & \kappa & \ldots & 0 \\
\ldots & \ldots & \ldots & \ldots & \ldots & \ldots \\
0 & \ldots & 0 & 0 & \kappa & 1
\end{array}\right]
$$

\section{G.4 Practical bisection method}

In this section we explore the performance of the bisection method we proposed in Section ?? of the main text. 

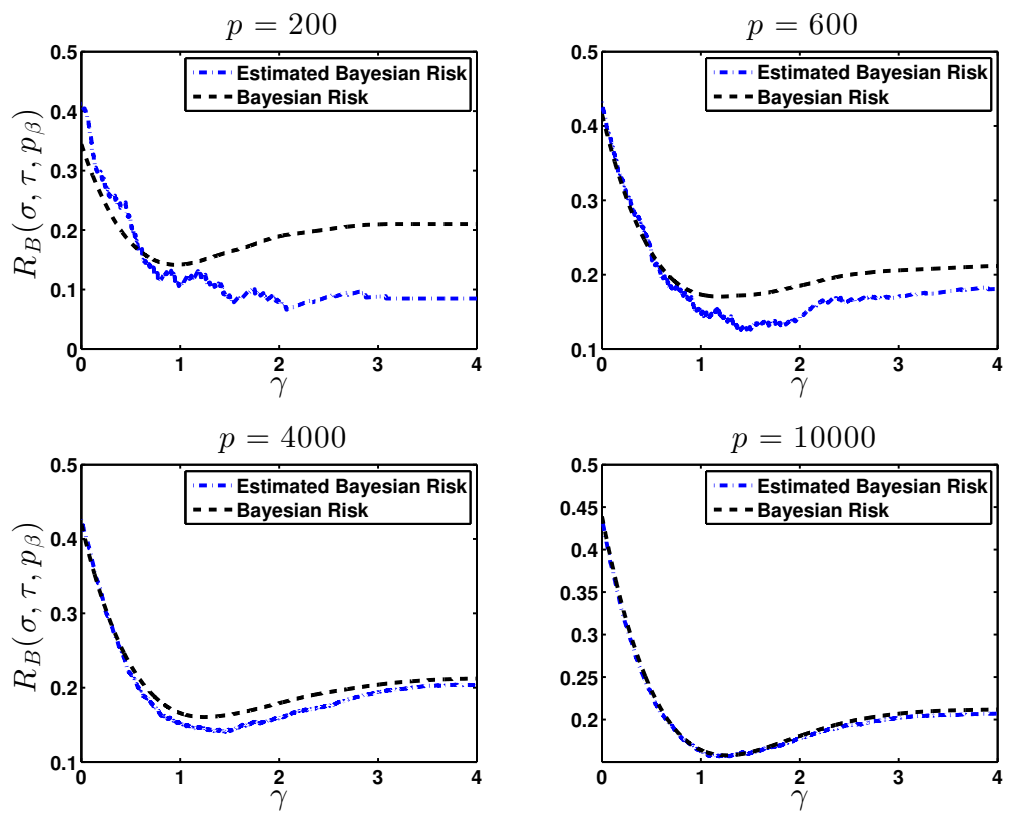

Figure 5: Performance of the risk estimator for different values of $p$ in the $10^{\text {th }}$ iteration of AMP. In this experiment $\delta=0.85, \rho=0.25$ and the standard deviation of measurement noise is 0.5. Black curve is the Bayes risk to which our estimates will converge as $p \rightarrow \infty$.

\section{G.4.1 Roadmap}

The bisection method introduced in Section ?? of the main text has the parameters $\epsilon$ and $\Delta, \bar{\tau}$, and $\underline{\tau}$. Setting these parameters under the asymptotic setting is straightforward; set $\epsilon, \Delta$ and $\underline{\tau}$ to a very small number, e.g. $10^{-10}$, and set $\tau$ to a large number. Under these settings the algorithm will work well in the limit $p \rightarrow \infty$. This is not necessarily true in medium problem sizes as we will describe later. Hence, before evaluating the performance of our algorithm on medium problem sizes, we should explain how these parameters are set. As we will show in the next few sections, setting these parameters is straightforward for even medium problem sizes. Sections G.5.1, G.5.2, and G.5.3 explore the impact of these parameters on the performance of the bisection algorithm and then present an automated method for setting these parameters. Section G.6 evaluates the performance of the automated bisection method on several problem instances. 

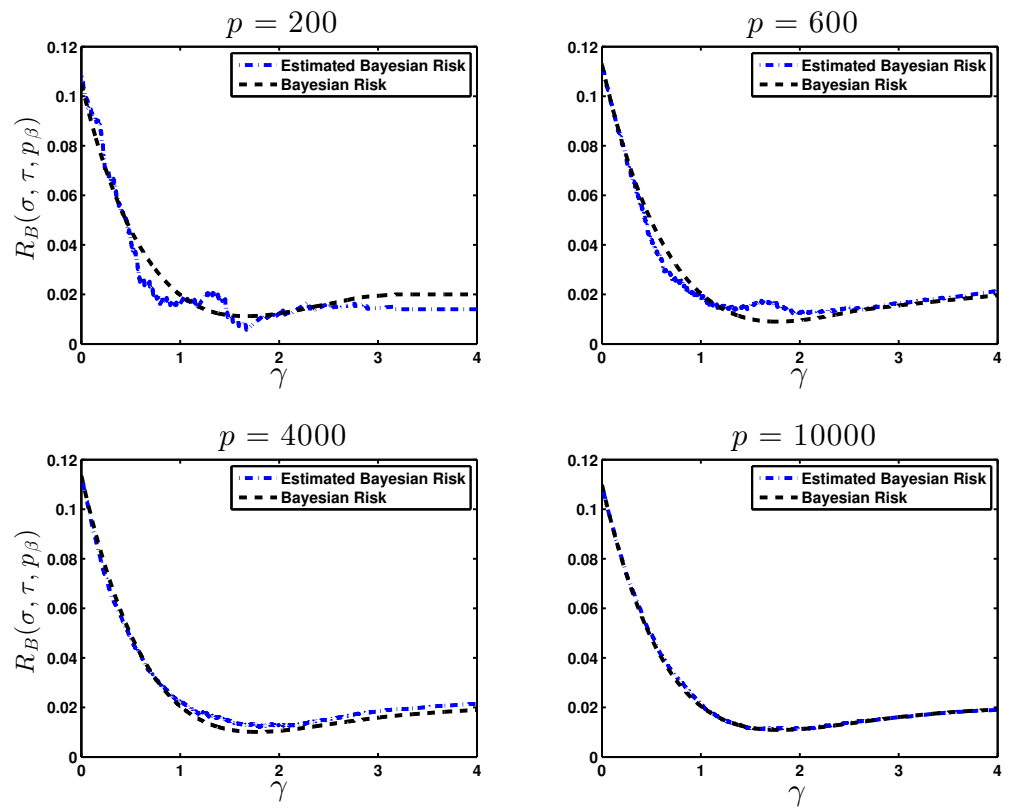

Figure 6: Performance of the risk estimator for different values of $p$ in the $1^{\text {st }}$ iteration of AMP. In this experiment $\delta=0.2, \rho=0.1$ and the standard deviation of measurement noise is 0.1. Black curve is the Bayes risk to which our estimates will converge as $p \rightarrow \infty$.

\section{G.5 Reparameterization}

A simple reparametrization of AMP may help us in setting the parameters of the bisection method. Define $\gamma^{t} \triangleq \tau^{t} / \sigma^{t}$. The problem of estimating the optimal value of $\tau^{t}$ is the same as that of $\gamma^{t}$ for $\sigma^{t}>0$. Furthermore, at iteration $t$ define $\bar{\gamma}=\bar{\tau} / \sigma$ and also $\underline{\gamma}=\underline{\tau} / \sigma$.

\section{G.5.1 Setting $\epsilon$}

As described in Section ?? of the main text we expect $\epsilon$ to be small. The exact value of $\epsilon$ impacts the number of iterations the bisection algorithm requires for convergence and also the accuracy of the bisection method. Since 15 iterations of bisection is sufficient for an accurate estimate of $\gamma^{t}$ (note that at every iteration of the bisection method the size of the search interval is halved), we can set the number of iterations of the bi-section method to 15 and set $\epsilon$ to 0 . If we want to reduce the number of iterations of the bisection method, then we can pick larger values of $\epsilon$. However, note that each iteration of the bisection method is in-expensive and whether we do it for 10 or 15 iterations does not affect the overall computational complexity of the algorithm much. 

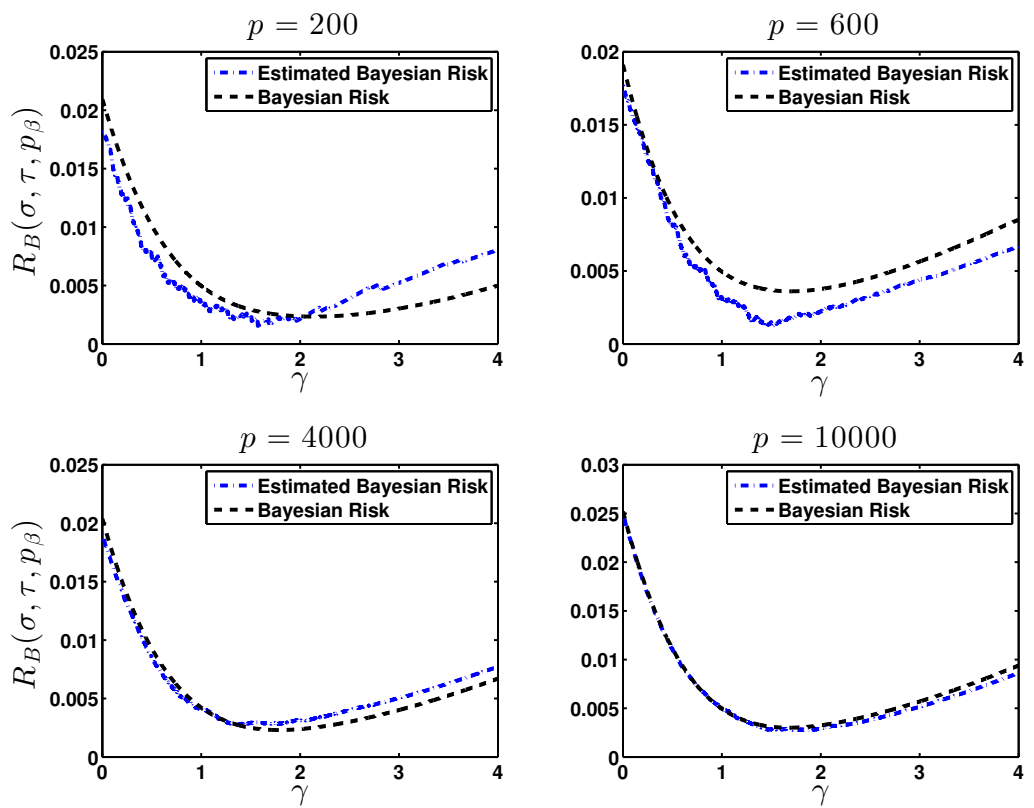

Figure 7: Performance of the risk estimator for different values of $p$ in the $10^{\text {th }}$ iteration of AMP. In this experiment $\delta=0.2, \rho=0.1$ and the standard deviation of measurement noise is 0.1. Black curve is the Bayes risk to which our estimates will converge as $p \rightarrow \infty$.

\section{G.5.2 Setting $\Delta$}

The second parameter of the bisection method is $\Delta$. While asymptotically we want $\Delta$ to be very small, in finite sample sizes this is slightly more tricky. If $\Delta$ is too small, then our estimate of the derivative of $\hat{R}$, introduced in Section ?? of the main text, will not be accurate and hence the algorithm may be trapped at a point far from the optimal value of $\tau^{t}$. Figure 13 shows this phenomenon. This figure shows the performance of the bisection method for 6 different values of $\Delta$. As is clear in this figure, the algorithm fails when $\Delta$ is too small ( $\left.\Delta=10^{-3}\right)$ or too large $(\Delta>1)$. However, for a wide range of values of $\Delta$, i.e. from $\Delta=0.01$ to $\Delta=1$, the algorithm performs well. Also, note that as we increase the dimension, the range of values of $\Delta$ for which the algorithm works well expands as predicted by our asymptotic results. This phenomenon can be observed by comparing Figures 13 and 14 and is due to the fact that our risk estimate becomes more accurate as $p$ increases. Furthermore, remember that the main reason we preferred the bisection method over the grid search in the AMP tuning was the fact that we have to apply it at every iteration of AMP. If we wanted to apply it in only one iteration, then we could have done a grid 

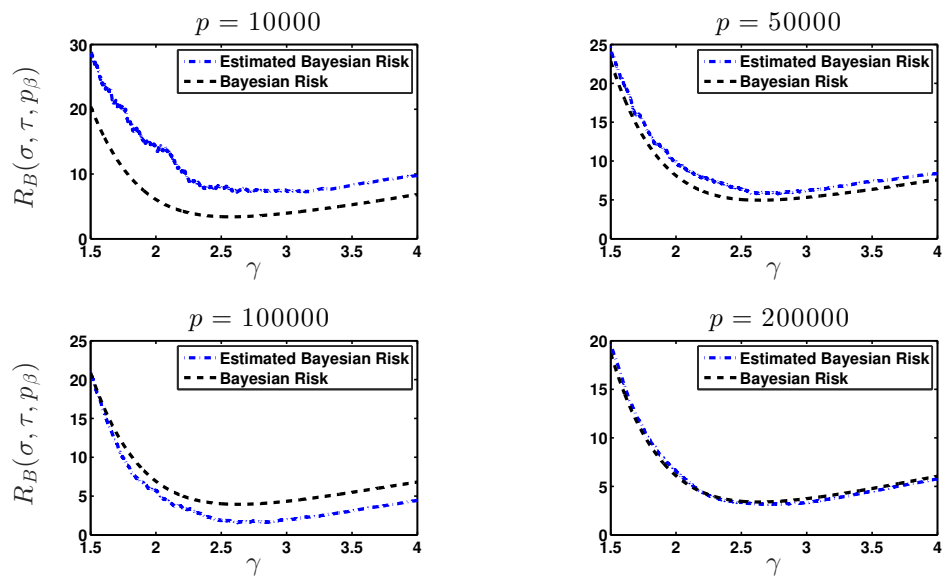

Figure 8: Performance of the risk estimator for different values of $p$ in the $10^{\text {th }}$ iteration of AMP. In this experiment $\delta=0.01, \rho=0.1$, and we consider noiseless measurements. We consider a point mass at 100 for the distribution of non-zero entries of $\beta_{o}$. Black curve is the Bayes risk to which our estimates will converge as $p \rightarrow \infty$.
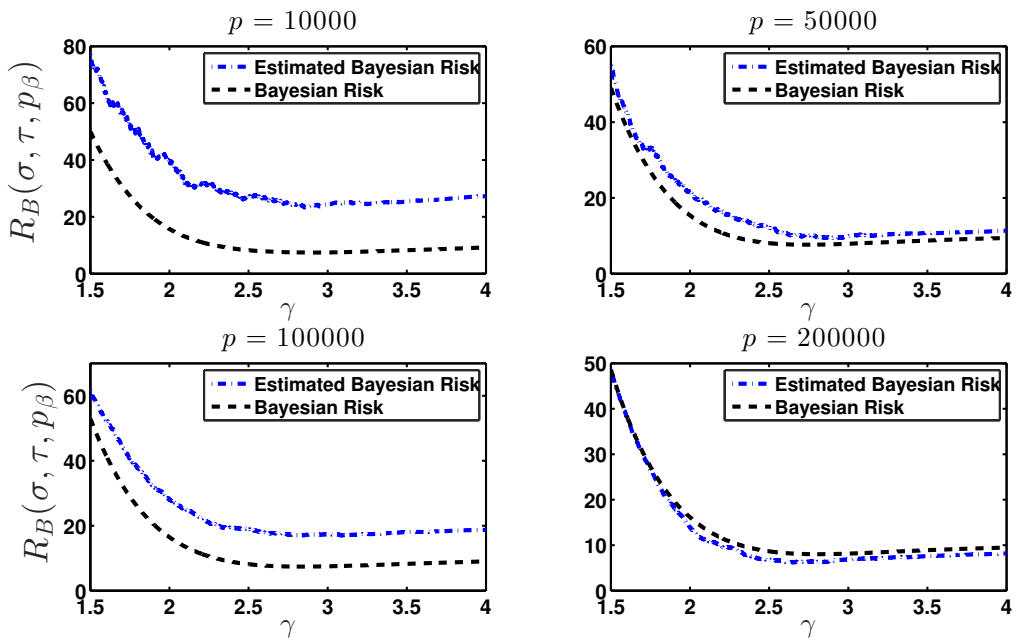

Figure 9: Performance of the risk estimator for different values of $p$ in the $1^{\text {st }}$ iteration of AMP. In this experiment $\delta=0.01, \rho=0.1$, and we consider noiseless measurements. We consider a point mass at 100 for the distribution of non-zero entries of $\beta_{o}$. Black curve is the Bayes risk to which our estimates will converge as $p \rightarrow \infty$. 

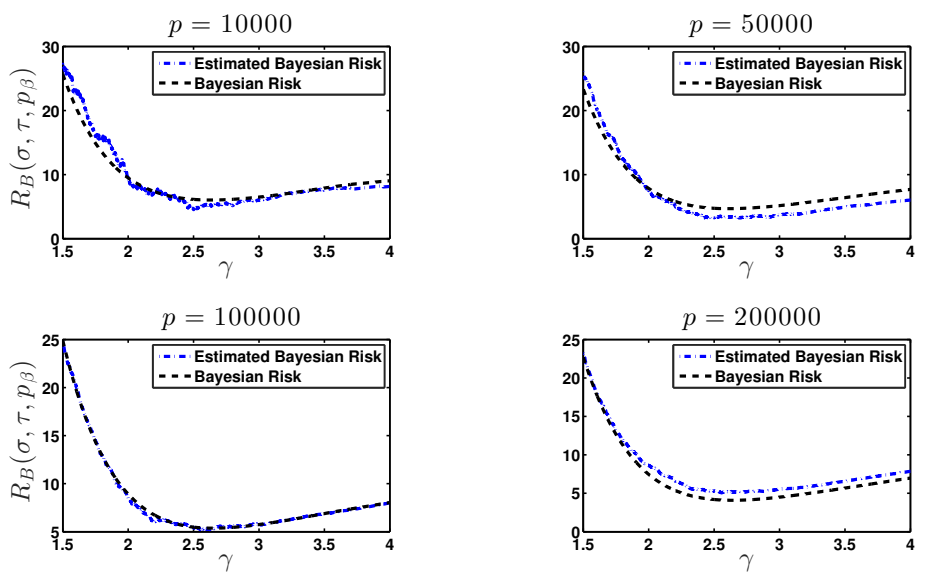

Figure 10: Performance of the risk estimator for different values of $p$ in the $10^{\text {th }}$ iteration of AMP. In this experiment $\delta=0.01, \rho=0.1$, and the standard deviation of measurement noise is 0.1 . We consider a point mass at 100 for the distribution of non-zero entries of $\beta_{o}$. Black curve is the Bayes risk to which our estimates will converge as $p \rightarrow \infty$.
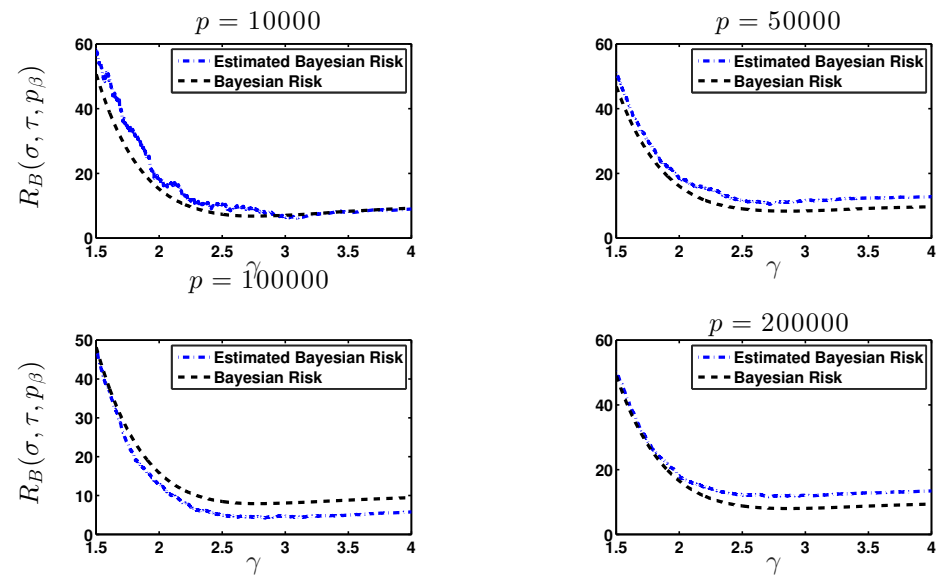

Figure 11: Performance of the risk estimator for different values of $p$ in the $1^{\text {st }}$ iteration of AMP. In this experiment $\delta=0.01, \rho=0.1$, and the standard deviation of measurement noise is 0.1 . We consider a point mass at 100 for the distribution of non-zero entries of $\beta_{o}$. Black curve is the Bayes risk to which our estimates will converge as $p \rightarrow \infty$. 


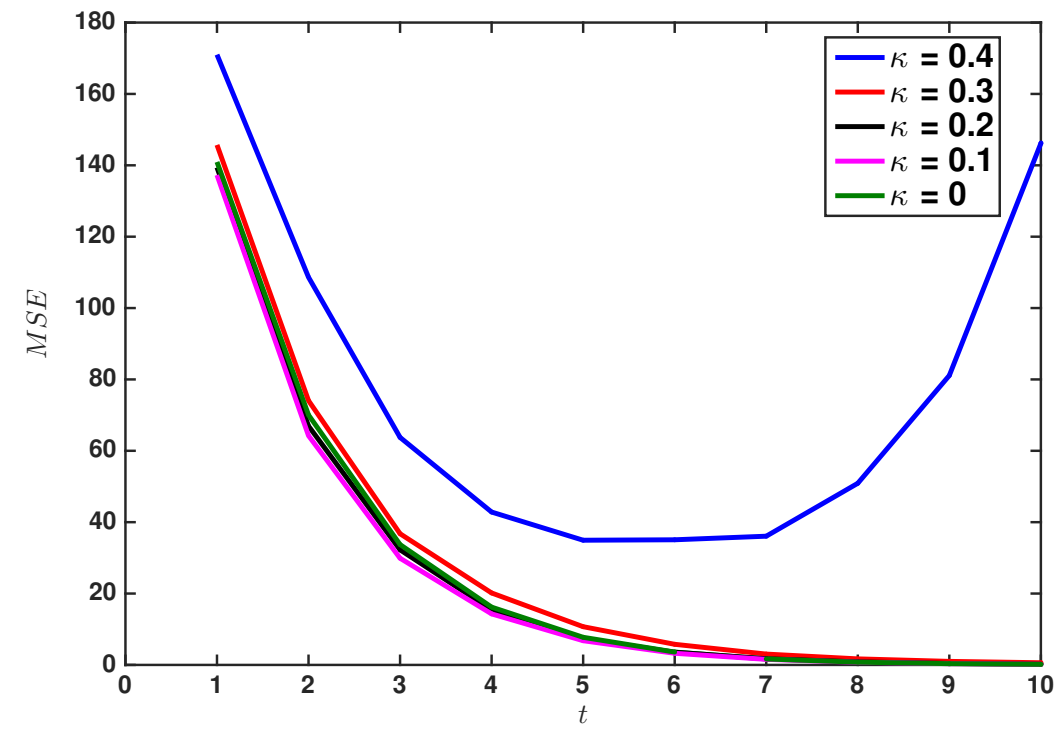

Figure 12: Performance of AMP tuned with modified bisection algorithm for different correlated measurement matrix design. $\kappa$ is a parameter in the covariance matrix of normal distribution from which rows of measurement matrix are sampled as noted in (45). In this experiment $p=10000, \delta=0.3, \rho=0.1$, and measurements are noiseless. We consider a point mass at 100 for the distribution of non-zero entries of $\beta_{o}$.

search. This motivates the following approach for setting $\Delta$ : Set $\Delta$ accurately in the first iteration and use the same $\Delta$ for the other iterations of AMP. Hence, we do the following steps for setting $\Delta$.

i. Set $\Delta$ according to the following procedure in the first iteration. For every $\Delta \in \mathcal{D}=\left\{10^{-\ell_{1}}, 5 \times 10^{-\ell_{1}+1}, \ldots, 5^{\ell_{2}} \times 10^{-\ell_{1}}\right\}$. Typical values for $\ell_{1}$ and $\ell_{2}$ are -5 and 6 respectively. Run the bisection method for the first iteration of AMP with these different values. Pick the one that leads to the best $\gamma$, i.e., the one that has the minimum risk estimate. Call this value of $\Delta, \Delta^{*}$.

ii. Use $\Delta^{*}$ for all the other iterations of AMP.

Note that the computational complexity of this approach is in general less than six times that of a bisection method in the first iteration and is the same for the other iterations. Hence, it does not have a major effect on the overall computational complexity of AMP. 

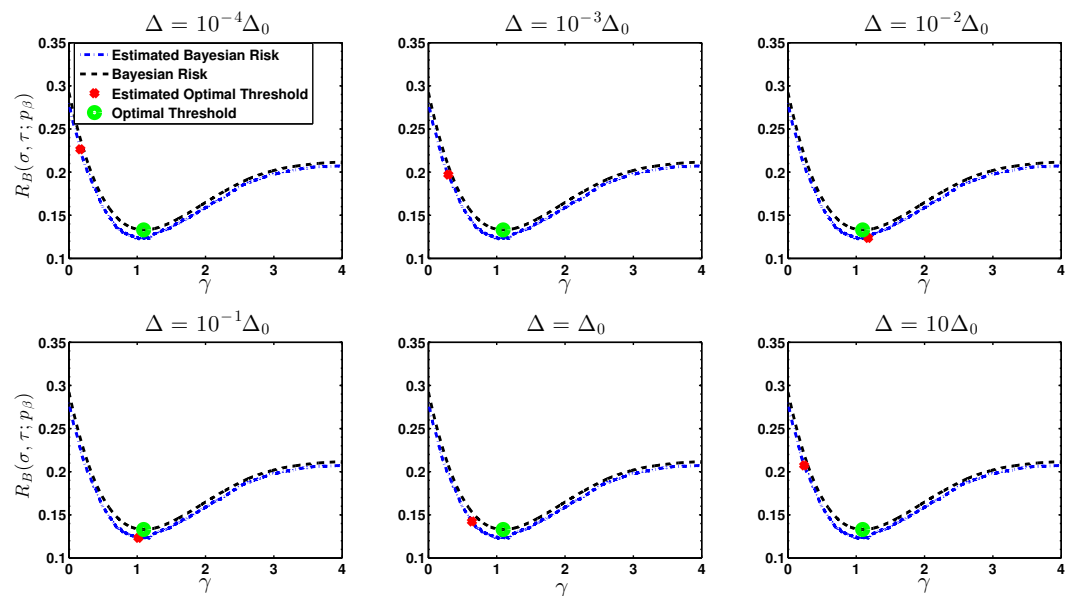

Figure 13: Performance of modified bisection algorithm in estimating $\hat{\gamma}_{o p t}$ for different values of $\Delta$. In this experiment $p=4000, \delta=0.85, \rho=0.25$, and the standard deviation of the noise of the measurements $\sigma$ is 0.2 . Blue dashed curve and black dashed curve show the estimated and the true risk functions, respectively. Green circle shows the optimal threshold. Finally, the red cross shows the estimated optimal threshold.

\section{G.5.3 Setting $\bar{\gamma}$ and $\gamma$}

Setting these two parameters is also straightforward. $\gamma \geq 0$. Hence, we set it to 0 . Also we set $\bar{\gamma}=\sup _{i}\left|\left(\beta^{t}+X^{*} z^{t}\right)_{i}\right| / \sigma^{t}$. Note that any value of $\gamma>\bar{\gamma}$ leads to zero estimate and will hence give the same estimate as $\bar{\gamma}$.

\section{G.6 Performance of the practical bisection method}

\section{G.6.1 Summary of the bisection method}

Our practical bisection method is summarized in the following flowchart. In this section we evaluate the quality of the estimates obtained from the modified bisection algorithm at different iterations of AMP. We use following experimental setup outlined in Algorithm 1.

(i) We set $p=2000$ in all experiments unless we mention otherwise. Number of measurements $n$ and the level of sparsity $k$ are obtained according to $n=\lfloor\delta p\rfloor$ and $k=\lfloor\rho n\rfloor$. In these experiments we set $\delta=0.85$ and $\rho=0.25 . \quad X$ is a measurement matrix having iid entries drawn from Gaussian distribution $N\left(0, \frac{1}{n}\right)$. The signal to be reconstructed $\beta_{o} \in \mathbb{R}^{p}$ has only $k$ non-zero values. We have tested the performance on several distributions for the non-zero entries of $\beta_{o}$. However, in the following 

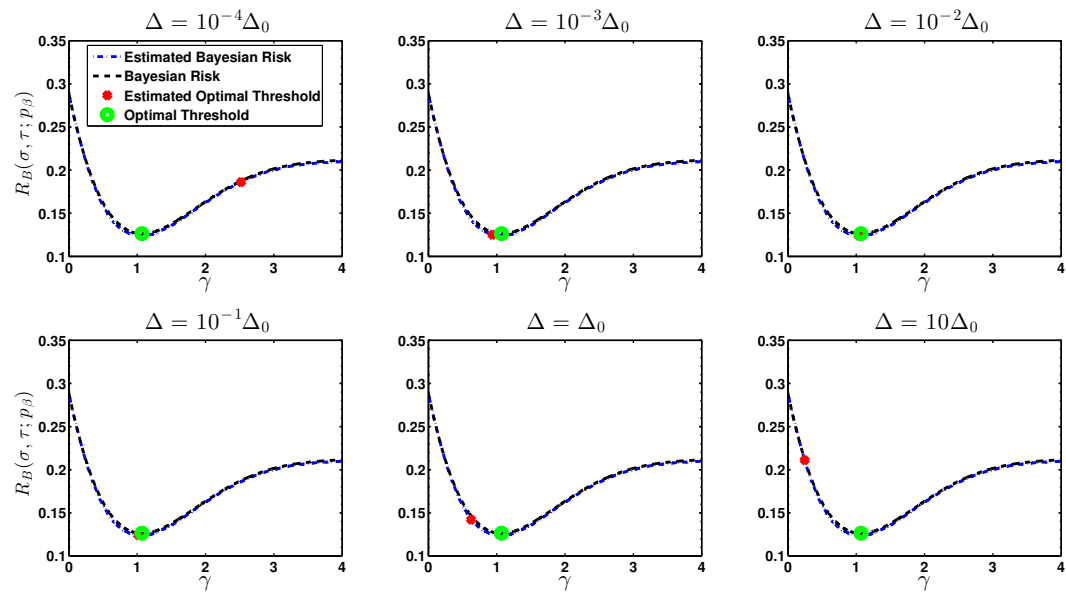

Figure 14: Performance of modified bisection algorithm in estimating $\hat{\gamma}_{o p t}$ for different values of $\Delta$. In this experiment $p=40000, \delta=0.85, \rho=0.25$, and the standard deviation of the noise of the measurements $\sigma$ is 0.2 . Blue dashed curve and black dashed curve show the estimated and true risk functions, respectively. Green circle shows the optimal threshold. Finally, the red cross shows the estimated optimal threshold.

experiments we consider a point mass at 1 for the distribution for nonzero entries of $\beta_{o}$.

(ii) The maximum number of iterations of AMP is set to 200. One can study other stopping rules to improve the efficiency of the algorithm. The measurements are given by $y=X \beta_{o}+w$, where $w \sim N\left(0, \sigma_{w}^{2} I\right)$. We consider 2 different cases for the noise: $\sigma_{w}=0,0.2$.

Figure 15 shows the performance of modified bisection algorithm for a set of noise-free measurements, i.e. $\sigma_{w}=0$. It contains 4 plots each of which corresponds to a specific iteration of AMP. Each plot in Figure 15 contains the Bayesian risk (defined in (??) of the main text), estimate of the Bayesian risk, the estimate of the optimal value of $\gamma$ obtained from the bisection method, $\hat{\gamma}_{o p t}$, and the optimal value of $\gamma, \gamma_{\text {opt }}$.

We now add some measurement noise to our system and set $\sigma_{w}=0.2$ and repeat the same experiment. Figure 16 summarizes our simulation result.

\section{G.6.2 Comparison with other tuning approaches of AMP}

Now we compare the performance of our proposed algorithm (modified bisection method for AMP tuning) with other tuning approaches proposed for AMP. There are two main approaches that have been proposed elsewhere: (i) maximin tuning that picks the parameter that achieves the highest phase transition $[2,7]$, 

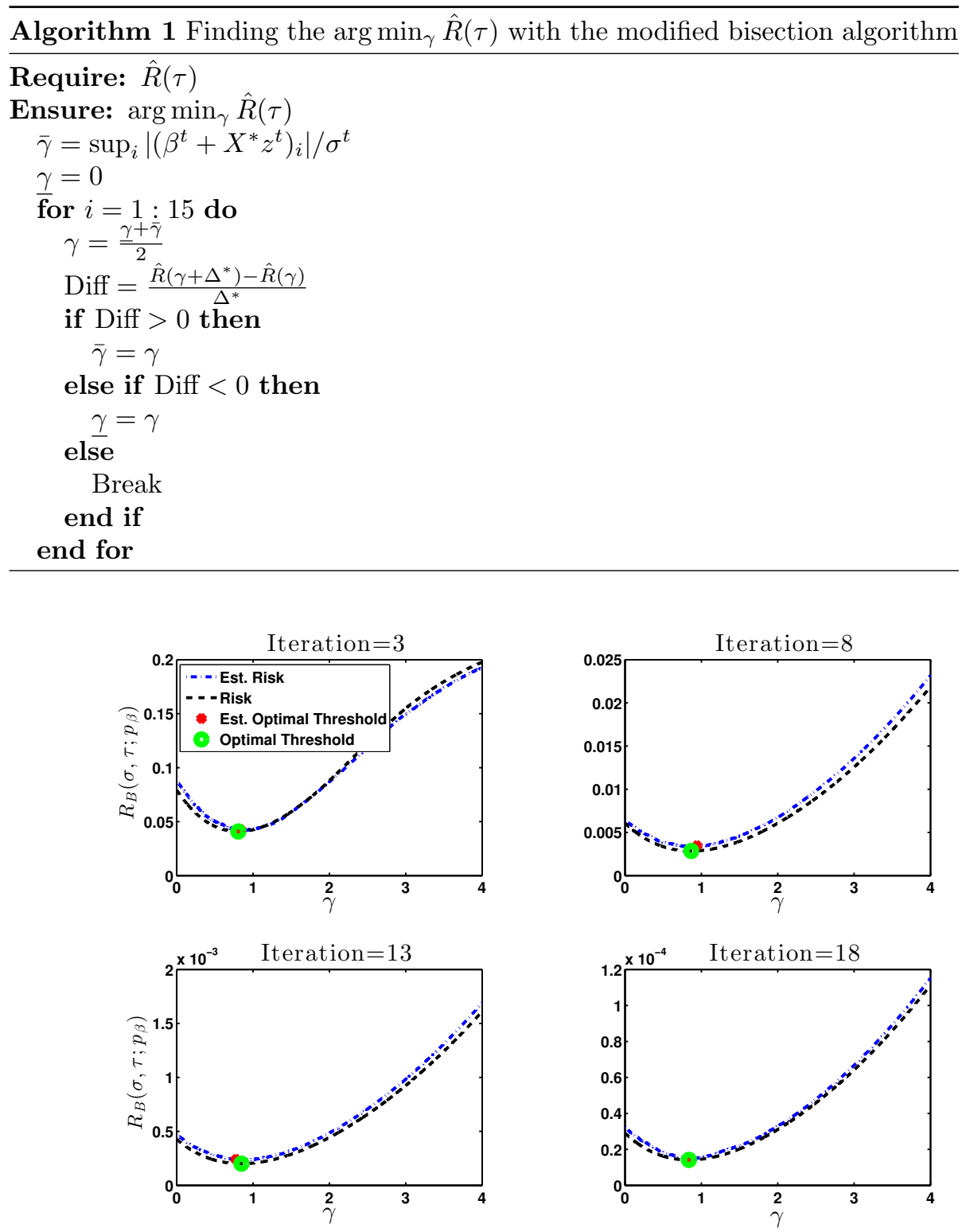

Figure 15: Performance of modified bisection method in estimating $\hat{\gamma}_{o p t}$ in different iterations of AMP. In this experiment $p=2000, \delta=0.85, \rho=0.25$, and we consider noiseless measurements.

and (ii) minimax tuning that selects the parameter that achieves the lowest noise sensitivity [3]. Among these two approaches the second approach is not practical 

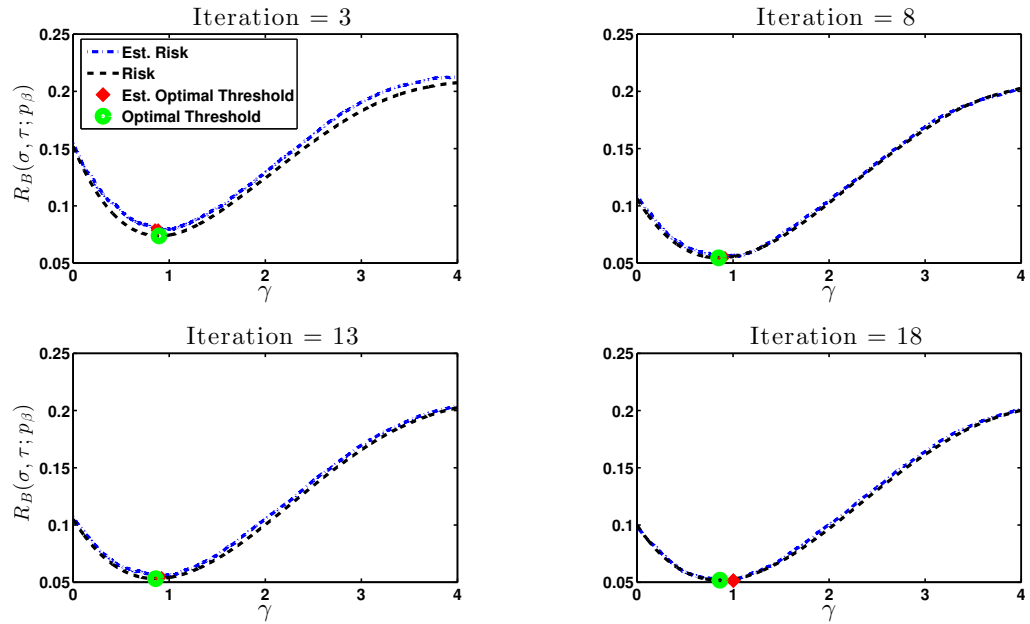

Figure 16: Performance of modified bisection method in estimating $\hat{\gamma}_{\text {opt }}$ in different iterations of AMP. In this experiment $p=2000, \delta=0.85, \rho=0.25$, and the standard deviation of the noise of the measurements $\sigma$ is 0.2 .

since it requires the sparsity level of $\beta_{o}$. Furthermore, to be optimal for any signal, it requires to know the distribution of the non-zero coefficients of $\beta_{o}$ as well. Hence, while the second method is a useful tool for theoretical analysis it cannot be considered a practical parameter tuning scheme. On the other hand, the maximin method is a method of tuning for the noiseless settings and has shown good performance in simulations [2]. In this section, we compare the performance of our algorithm with the maximin approach under the noiseless setting (the setting in which maximin is designed for) to show that even in such cases our approach provides a better convergence rate than the maximin approach.

Figure 17 compares the convergence of the AMP for different tuning schemes. As we can see from the figures, the tuning scheme proposed in this paper is able to reconstruct the original signal up to a certain reconstruction error by the least number of iterations. This is much faster than the AMP that uses maximin tuning. For instance, the AMP with maximin tuning approach requires more than 17 iterations to achieve the accuracy that AMP tuned with modified bisection algorithm achieves in 10 iterations. Note that the extra calculations that the bisection method requires in 10 iterations, is much less than that of a one matrix-vector multiplication. Hence, overall with less computations we achieve more accurate results. Another advantage of AMP tuned with modified bisection algorithm over the AMP that is tuned according to maximin approach is that it adapts itself to the distribution of $\beta$, and the noise variance and will achieve the optimal MSE, while maximin AMP is only optimal for the noiseless settings. 
We should also note that although grid-search AMP performs almost like the AMP tuned with modified bisection algorithm, it needs an exhaustive search among many threshold values to find the optimal one and hence is computationally expensive.

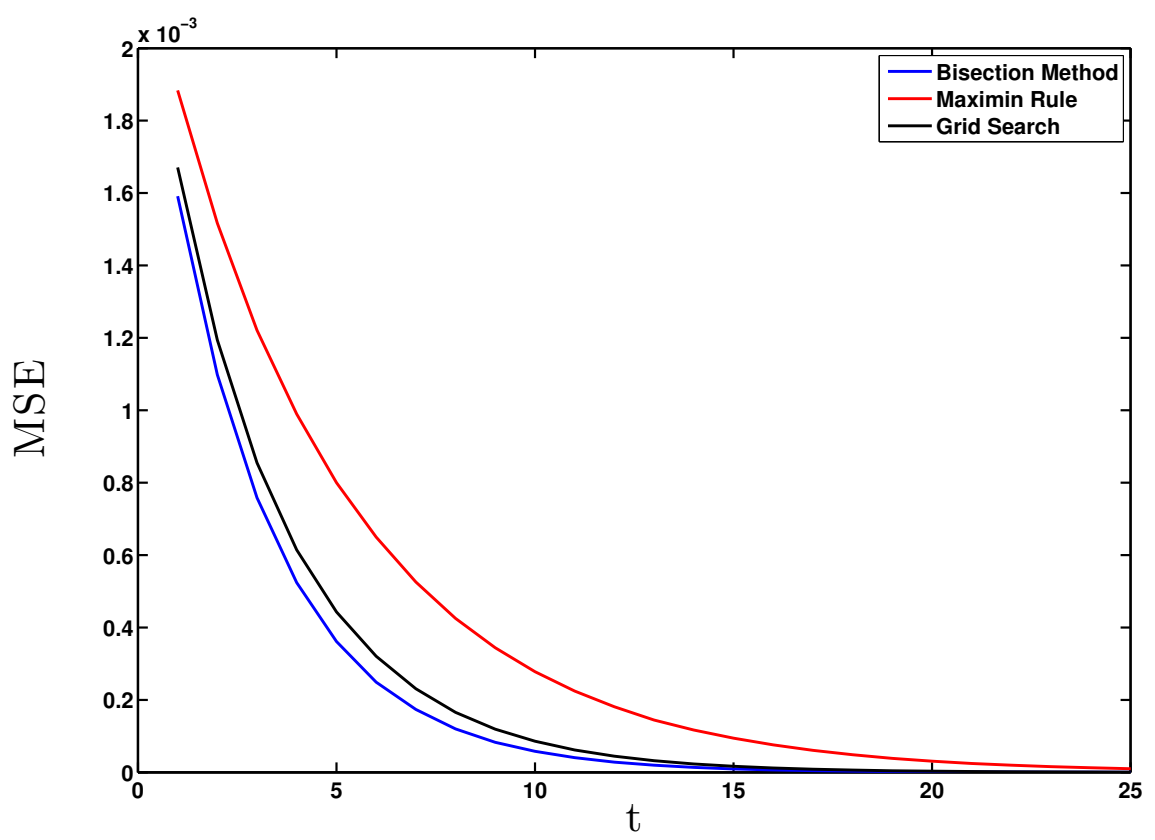

Figure 17: MSE of AMP at each iteration for three different threshold setting approaches. The blue curve corresponds to the modified bisection method introduced in this paper. In the black curve, the threshold is set to a constant value. This constant threshold, which causes the smallest reconstruction error, is found by the grid search method. In the red curve, threshold is set to the value which gives the best phase transition [3].

\section{G.7 Comparison of optimally-tuned AMP and LASSO}

Proposition 3.8 claims that as $t \rightarrow \infty$ the asymptotic mean square error of the optimally-tuned AMP converges to the asymptotic mean square error of LASSO. Our goal in this section is to first show through two simulations that this is the case, and also show how many iterations are in general required for the optimally-tuned AMP to reach the solution of LASSO. Toward this goal we solve LASSO for different values of the regularization parameter $\lambda$ and find the value of $\lambda$ that minimizes $\left\|\hat{\beta}^{\lambda}-\beta_{o}\right\|_{2}^{2}$. We consider 1000 evenly spaced points in the interval $\left[\lambda_{\min }, \lambda_{\max }\right]$ and solve LASSO for each of these regularization 
parameters. We then pick the $\lambda$ that minimizes $\left\|\hat{\beta}^{\lambda}-\beta_{o}\right\|_{2}^{2}$. Choosing $\lambda_{\min }$ and $\lambda_{\max }$ is straightforward. We set $\lambda_{\min }=0$ and choose $\lambda_{\max }$ such that $\left\|\hat{\beta}^{\lambda_{\max }}-\beta_{o}\right\|_{2}^{2} \sim\left\|\hat{\beta}^{\lambda_{\min }}-\beta_{o}\right\|_{2}^{2}$.

Suppose that $\tilde{\lambda}_{\text {opt }}$ denotes the optimal value of $\lambda$. Note that in our calculation of $\tilde{\lambda}_{\text {opt }}$ we have used $\beta$ which is not available in practice. We then compare $\left\|\hat{\beta}^{\tilde{\lambda}_{\mathrm{opt}}}-\beta_{o}\right\|_{2}^{2} / p$ with the $\left\|\beta^{t}-\beta_{o}\right\|_{2}^{2} / p$ where $\beta^{t}$ denotes the estimate of the optimally tuned AMP at time $t$. Figures 18 and 19 exhibit our comparisons in two different scenarios that are explained in the captions of the figures. We can see that in both figures the risk of our proposed algorithm converges to the minimum LASSO risk very fast.

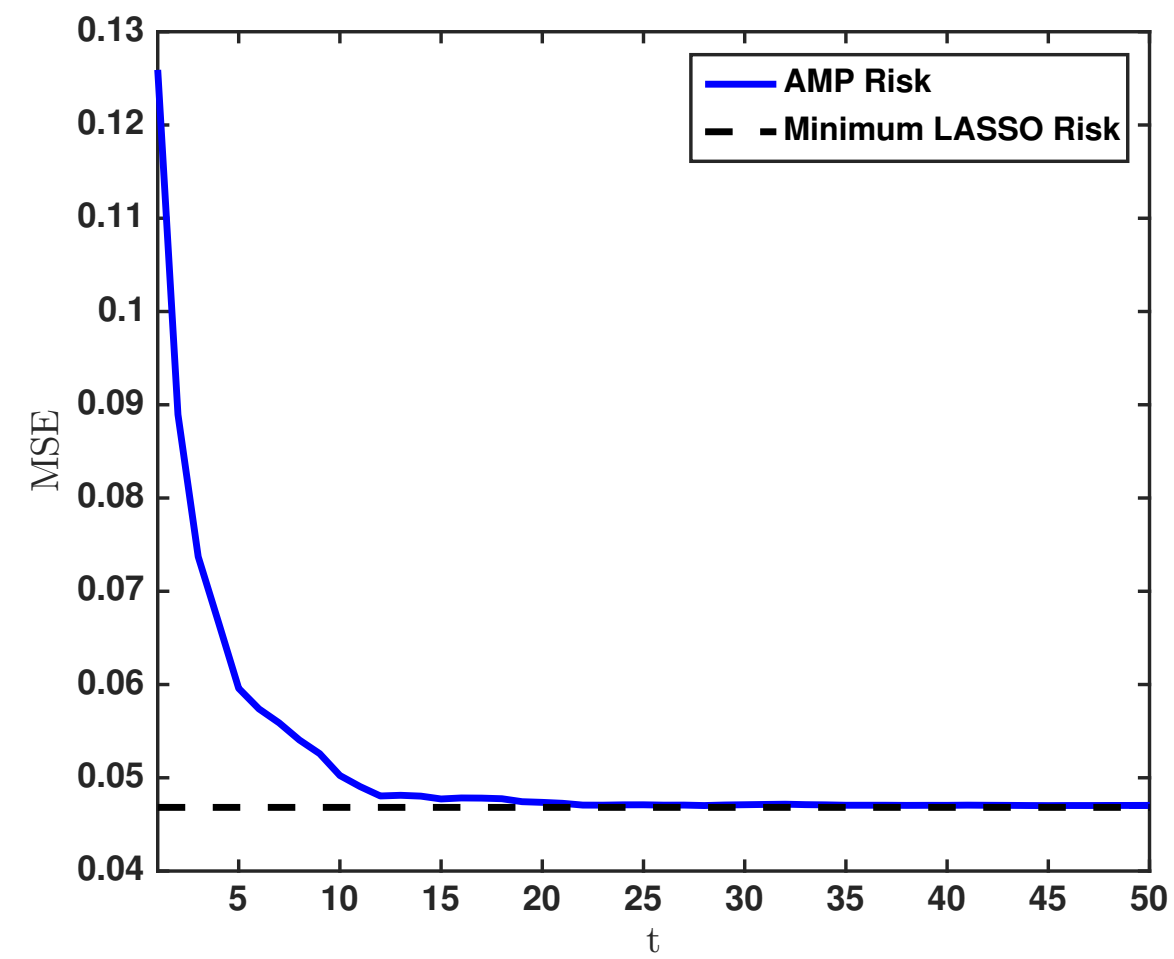

Figure 18: Comparing AMP risk (blue solid curve) with minimum LASSO risk (black dashed curve) over a grid of regularization parameters. In this experiment $p=2000, \delta=0.85, \rho=0.25$, and the standard deviation of measurement noise is 0.2 . 


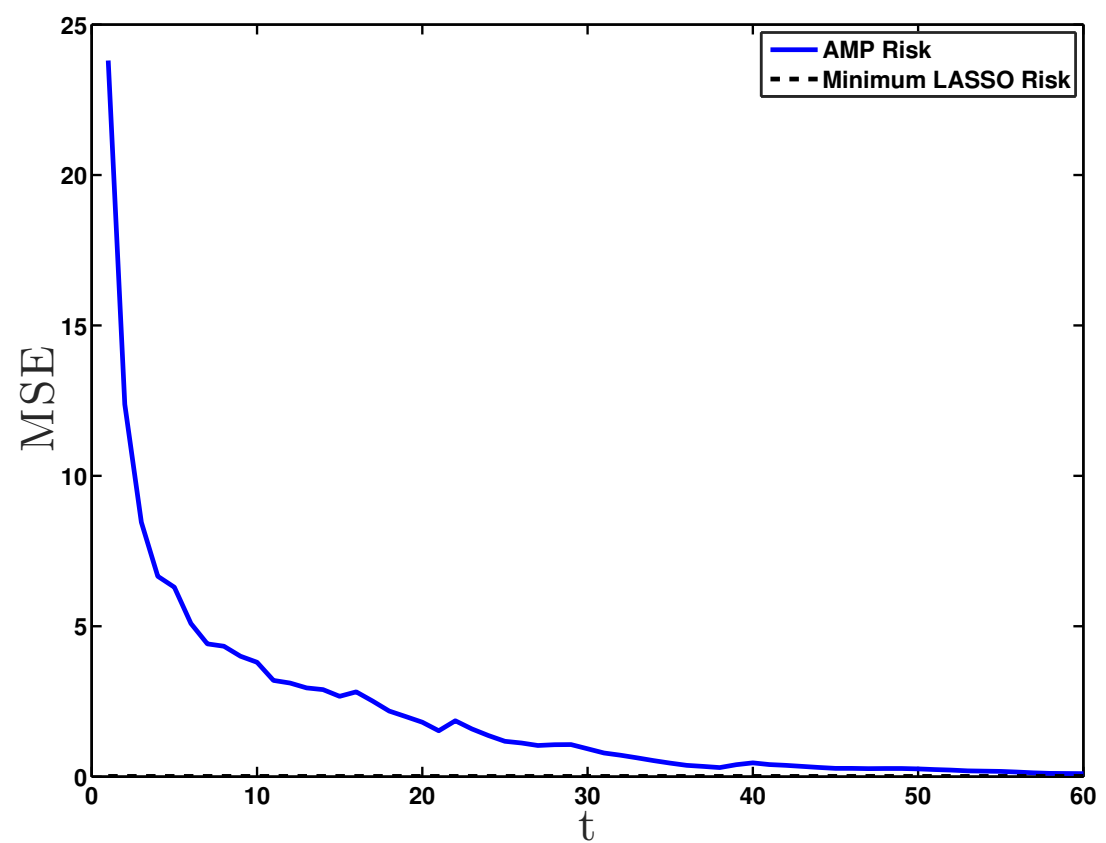

Figure 19: Comparing AMP risk (blue solid curve) with minimum LASSO risk (black dashed curve) over a grid of regularization parameters. In this experiment $p=10000, \delta=0.01, \rho=0.1$, and the standard deviation of measurement noise is 0.5 . We consider a point mass at 100 for the distribution of non-zero entries of $\beta_{0}$.

\section{G.8 Simulation Details}

Here we include the details of the simulations whose results we reported in the main text.

\section{G.8.1 Figure 20}

The dataset we used in this simulation is taken from [8]. The response variables $y \in \mathbb{R}^{442}$ correspond to the diabetes progression in one year in 442 patients. We have 10 variables, namely age, sex, body mass index, average blood pressure, and six blood serum measurements. Therefore $\beta \in \mathbb{R}^{10}$. We have solved LASSO for different values of $\lambda$ and presented the number of nonzero elements in $\beta$, i.e. $\|\beta\|_{0}$, as a function of $\lambda$ in Figure 20. As mentioned previously, for this specific problem, this function is not monotone decreasing. 


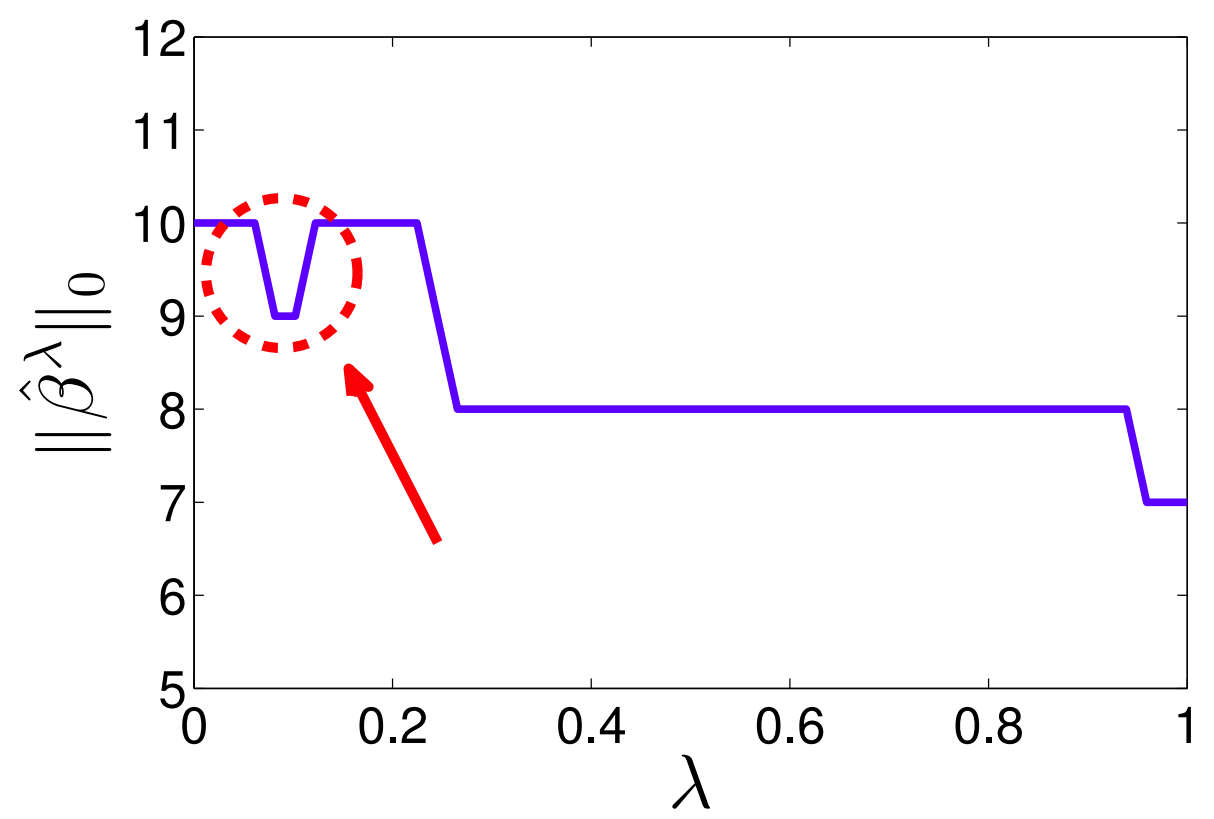

Figure 20: The number of active elements in the LASSO's solution as a function of $\lambda$. The size of the active set at one location grows as we increase $\lambda$ and hence this function does not match the intuition. For the details of this experiment, see Section G.8.1.

\section{G.8.2 Figure 21}

Here, we consider the recovery of a sparse signal in the presence of measurement noise. $\beta_{o} \in \mathbb{R}^{2000}$ is a sparse signal with 100 nonzero elements equal to 1 . We observe an undersampled noisy version of $\beta_{o}$, i.e., $y=X \beta_{o}+w$ where $X$ is a $1000 \times 2000$ matrix with iid entries having normal distribution $N(0,1)$, and $w$ is a $1000 \times 1$ noise vector with iid entries having the Gaussian distribution $N(0,0.7)$. We consider 100 equi-spaced values of $\lambda$ between 0 and 0.25 . We then solve the LASSO and measure the $\left\|\hat{\beta}_{\lambda}\right\|_{0}$. Figure 21 represents the $\left\|\hat{\beta}_{\lambda}\right\|_{0}$ versus $\lambda$.

\section{G.8.3 Figures 22}

The setting here is the same as for Figure 21 with two differences. First, the $\lambda$ values are 100 equi-spaced values between 0 and 1. Second, the entries of $w$ are obtained from the Gaussian distributions $N(0,2)$ and $N(0,0.4)$. 


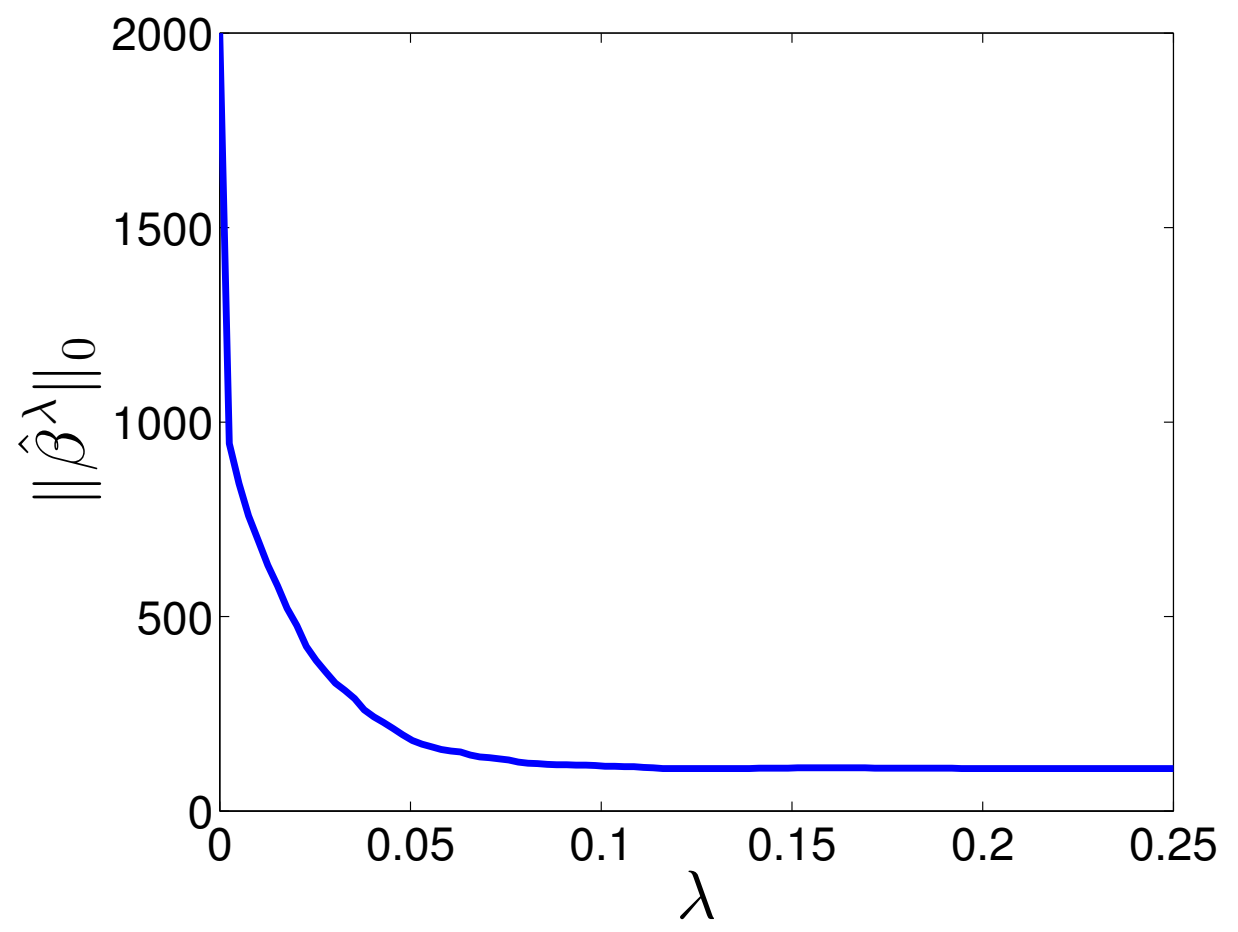

Figure 21: The number of active elements in the solution of LASSO as a function of $\lambda$. The size of the active set decreases monotonically as we increase $\lambda$. See Section G.8.2 for the details.

\section{References}

[1] S. Boyd and L. Vanderberghe. Convex Optimization. Cambridge University Press, 2004.

[2] D. L. Donoho, A. Maleki, and A. Montanari. Message passing algorithms for compressed sensing. Proc. Natl. Acad. Sci., 106(45):18914-18919, 2009.

[3] D. L. Donoho, A. Maleki, and A. Montanari. Noise sensitivity phase transition. IEEE Trans. Inform. Theory, 57(10):6920-6941, Oct. 2011.

[4] Yong-Qua Yin, Zhi-Dong Bai, and Pathak R Krishnaiah. On the limit of the largest eigenvalue of the large dimensional sample covariance matrix. Probability theory and related fields, 78(4):509-521, 1988.

[5] M. Bayati and A. Montanri. The dynamics of message passing on dense graphs, with applications to compressed sensing. IEEE Trans. Inform. Theory, 57(2):764-785, Feb. 2011. 

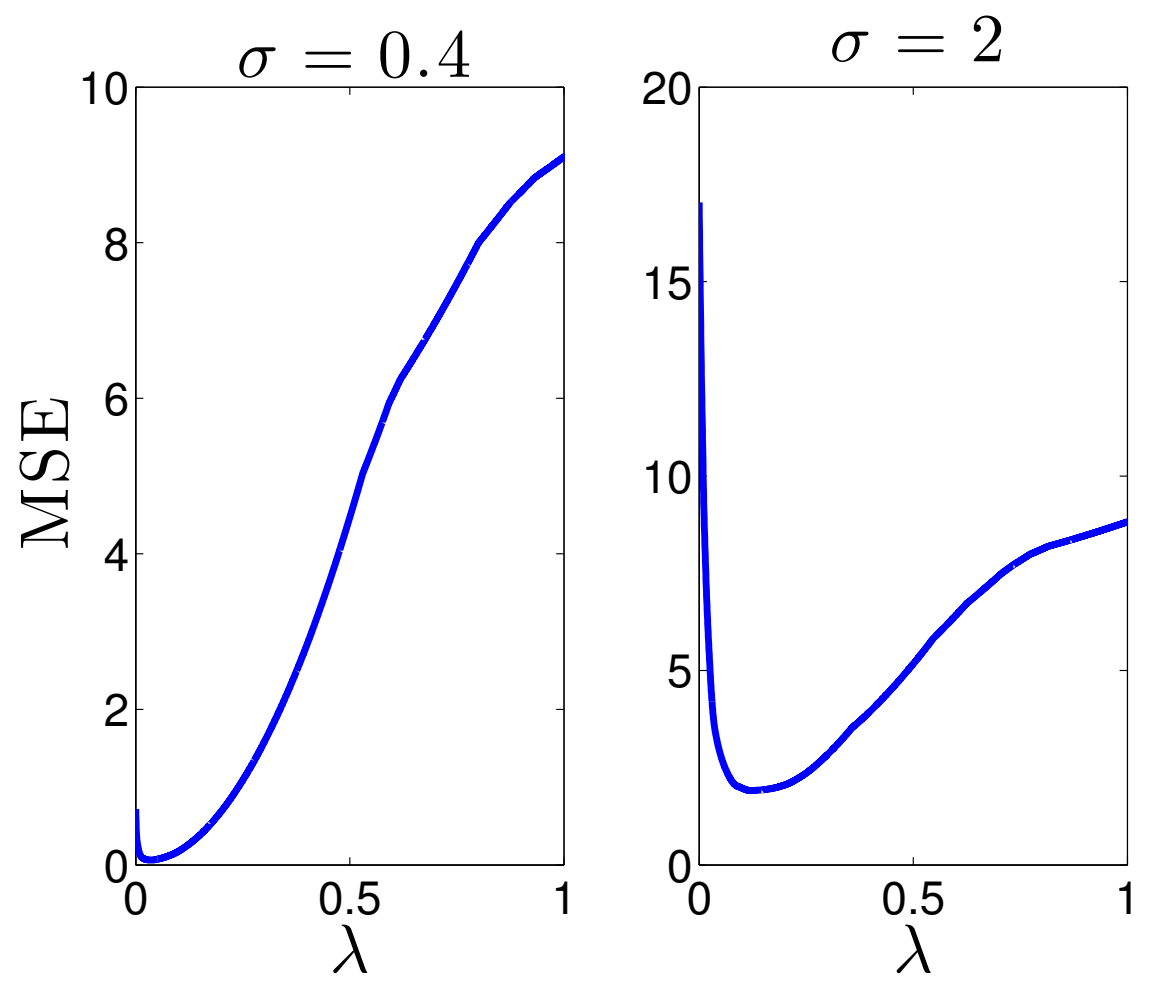

Figure 22: Behavior of the MSE as a function of $\lambda$ of LASSO for two different noise variances. See Section G.8.3 for details. 
[6] M. Bayati and A. Montanari. The LASSO risk for Gaussian matrices. Information Theory, IEEE Transactions on, 58(4):1997-2017, 2012.

[7] A. Maleki and D. L. Donoho. Optimally tuned iterative thresholding algorithm for compressed sensing. IEEE J. Select. Top. Signal Processing, 4(2):330-341, April 2010.

[8] B. Efron, T. Hastie, I. Johnstone, and R. Tibshirani. Least angle regression. Ann. Stat., 32(2):407-499, 2004. 